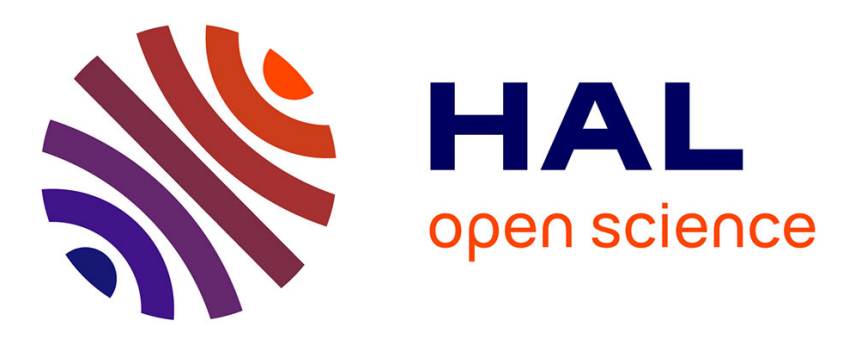

\title{
Identification and Extraction of Surface Waves from Three-component Seismograms based on the Normalized Inner Product
}

Kristel Carolina Meza Fajardo, Apostolos Papageorgiou, Jean-François Semblat

\section{To cite this version:}

Kristel Carolina Meza Fajardo, Apostolos Papageorgiou, Jean-François Semblat. Identification and Extraction of Surface Waves from Three-component Seismograms based on the Normalized Inner Product. Bulletin of the Seismological Society of America, 2015, 105 (1), pp.210-229. 10.1785/0120140012 . hal-02147535

\section{HAL Id: hal-02147535 \\ https://hal.science/hal-02147535}

Submitted on 4 Jun 2019

HAL is a multi-disciplinary open access archive for the deposit and dissemination of scientific research documents, whether they are published or not. The documents may come from teaching and research institutions in France or abroad, or from public or private research centers.
L'archive ouverte pluridisciplinaire HAL, est destinée au dépôt et à la diffusion de documents scientifiques de niveau recherche, publiés ou non, émanant des établissements d'enseignement et de recherche français ou étrangers, des laboratoires publics ou privés. 
1 TITLE:

2 Identification and Extraction of Surface Waves from Three-component Seismograms based on the

3 Normalized Inner Product

4

5 AUTHORS' NAMES:

6 Kristel C. Meza-Fajardo ${ }^{(1)}$, Apostolos S. Papageorgiou ${ }^{(2)}$ and Jean-François Semblat ${ }^{(3)}$

7

8 CORRESPONDING AUTHOR:

$9 \quad$ Kristel C. Meza-Fajardo

10 Department of Civil Engineering,

11 Universidad Nacional Autónoma de Honduras

12 Tegucigalpa, Honduras

13 E-mail: kristelmeza@unah.edu.hn

14

15 
17 Identification of different wave types in a seismogram is an important step for the understanding of

18 wave propagation phenomena. Since in most seismograms, different types of waves with different

19 frequencies may appear simultaneously, separation of waves is more effectively achieved when a time-

20 frequency analysis is performed. In this work, we propose a new time-frequency analysis procedure to

21 identify and extract Rayleigh and Love waves from three-component seismograms. Exploiting the

22 advantage of the absolute phase preservation by the Stockwell Transform, we construct time-frequency

23 filters to extract waves based on the 'Normalized Inner Product' (NIP). Since the NIP is the time-

24 frequency counterpart of the correlation, Rayleigh and Love waves can be identified depending on the

25 NIP between the Stockwell Transforms of the horizontal and vertical displacement components. The

26 novelty and advantage of the proposed procedure is that it does not require specifying a-priori the

27 direction of propagation of the surface waves, but instead such direction is determined. Furthermore, it is

28 shown that the NIP is a more stable parameter in the time-frequency domain when compared to the

29 instantaneous reciprocal ellipticity, and thus it avoids smoothing (and with it, altering) the data. The

30 procedure has been successfully tested with real signals, specifically to extract Rayleigh and Love waves

31 from seismograms of one aftershock of the 1999 Chi-Chi earthquake. With the proposed procedure we

32 found different directions of propagation for retro-grade and pro-grade Rayleigh waves, which might

33 suggest that they are generated by different mechanisms. 


\section{INTRODUCTION}

35 The identification of surface waves in a time history is one of the fundamental tasks in seismology.

36 Surface waves are important not only because they carry information about the surficial geological

37 layers in which they propagate, but also for their impact on man-made structures. Methods for

38 identifying surface waves are based on the two main characteristics of such waves: (1) plane and type of

39 polarization, and (2) frequency-dependent phase velocities (dispersion). A processor to identify surface

40 waves and simultaneously compute their azimuth, was proposed in the late 70's by Smart (1978). The

41 algorithm finds the best fit between polarization characteristics of ground motion and surface wave

42 models defined in the frequency domain. More recently, identification of Rayleigh wave phases has

43 been performed by means of Complex Trace Analysis (CTA) (Vidale, 1986, René et al., 1986, Li and

44 Crampin, 1991, Baker and Stevens, 2004). CTA uses time-varying polarization characteristics to

45 differentiate between waves, and thus, waves arriving at different times can be separated. Rayleigh

46 waves are identified by considering the fact that they are elliptically polarized in a plane oriented in their

47 direction of propagation. However, in view of the fact that dispersed waves (such as surface waves) may

48 be effectively described and analyzed in terms of narrow-band wave packets, we need an extraction

49 technique that resolves the recorded signals in such narrow-band packets. Since CTA does not provide

50 information on the time variation of the frequency content of the signal, the analyst needs to choose

51 frequency ranges of interest a priori. This problem is aggravated when different types of waves appear

52 simultaneously in the signal under investigation, as it often happens with seismic waves. Another

53 difficulty faced by the analyst is the need to assume a priori the direction of propagation of the surface

54 waves present in the time histories. 
55 Considering the above reasons, a time-frequency polarization analysis seems to be a more appropriate

56 alternative in order to separate the different phases in a wave field. Whereas most of classical signal

57 processing studies of the 1970s were aimed at stationary signals and processes, many efforts were

58 devoted to less idealized situations during the 1980s, and the idea of time-frequency analysis

59 progressively emerged as a new paradigm for non-stationarity. It is now well recognized that many

60 signal processing problems can be advantageously phrased in a time-frequency language. Pinnegar

61 (2006) and Galiana-Merino et al. (2011) have constructed filters to exclude or extract Rayleigh waves

62 using their elliptical polarization as filtering criterion. However, filtering based on the elliptical

63 polarization attribute alone does not work well if the time history contains Rayleigh waves with both

64 retro-grade and pro-grade motion. Such a case has been observed in recordings of the aftershock 1803 of

65 the Chi-Chi earthquake that occurred on 20 September 1999 with magnitude Mw 6.2 (Wang et al.,

66 2006). The method we propose herein to detect and extract surface waves from three-component

67 recorded seismograms overcomes these difficulties. We exploit the advantage of the absolute phase

68 preservation of the Stockwell Transform (Stockwell et al., 1996), and we construct time-frequency

69 filters to extract waves based on the 'Normalized Inner Product' (NIP). Since the NIP is the time-

70 frequency counterpart of the correlation, Rayleigh and Love waves can be identified based on the value

71 of the NIP between the Stockwell Transforms of the horizontal and vertical displacement components.

72 The proposed procedure does not require specifying a-priori the direction of propagation of the surface

73 waves, but instead such direction is determined with the proposed computational procedure.

\section{TIME-FREQUENCY POLARIZATION ANALYSIS}

75 Polarization characteristics are useful tools to identify and separate different types of waves present in a 76 multi-component signal. If the components of the analyzed signal are in-phase, it is said that they are 
77 linearly polarized. When the components are 90 degrees out-of-phase and have the same amplitude, the 78 state corresponds to circular polarization. If the components are 90 degrees out-of-phase and have 79 different amplitudes, the state corresponds to elliptical polarization. Regarding waves contained in 80 seismic signals, the polarization state observed for Rayleigh waves is elliptical (either retro-grade or pro81 grade), whereas body and Love waves are considered to be linearly polarized.

82 Following the ideas presented by Pinnegar (2006), three-component signals can be thought as a 83 superposition of sinusoids oscillating along the -, -, and - axes, which when considered one

84 frequency at a time, trace out elliptical motion in 3D space. Thus, the total three component signal can 85 be thought of as a superposition of ellipses, which are characterized by descriptive parameters such as 86 the length of the semi-minor and semi-major axis, the strike and dip of each ellipse plane, the pitch of 87 the major axis, and the phase of the particle motion at each frequency. The Fourier spectra of the 88 descriptive parameters of the superimposed ellipses can be related to the Fourier transforms of the - , 89 and - components (Pinnegar, 2006). The same type of reasoning can be used with windowed Fourier 90 transforms (such as the Stockwell Transform), so as to provide time-varying spectra for the 91 abovementioned descriptive parameters. Details on how the attributes of the ellipses are defined for 92 three-component signals can be found in Pinnegar (2006).

93 In this work, we adopt the Stockwell Transform for the mapping between time and time-frequency 94 domains, because: 1) using the Stockwell Transform, we retain the absolute phase of each localized 95 frequency component (Stockwell et al., 1996), and 2) the invertibility of the Stockwell Transform allows 96 for the wave extraction by simple filtering in the time-frequency domain. The Stockwell Transform is a 97 generalization of the short-time Fourier transform (STFT), and may be thought of as an extension of the 98 continuous wavelet transform (CWT) while overcoming some of its disadvantages. It is based on a 
99 moving and scalable localizing Gaussian window, which features a standard deviation that is always 100 equal to one wavelength of the Fourier sinusoid (Pinnegar, 2006). The "moving window" technique has 101 been already used in the past in surface wave analyses (Flinn, 1965; Dziewonski et al., 1969; Vidale, 102 1986) based on the concept of the "analytical signal" of the time series. It can be shown (Stockwell, 103 2007) that the Stockwell Transform at a specific frequency is closely related to the analytical signal of 104 the time series, when the time series is bandpass filtered using the Gaussian window. The Stockwell 105 transform of a time varying function can be expressed in the following form (Stockwell et 106 al., 1996):

107 where is the center of the Gaussian window. The time-frequency parameters of the polarization ellipse, 108 which describe the contribution of the -th frequency to the total signal are defined in Pinnegar (2006) 109 in terms of the Stockwell transform. In particular, Pinnegar (2006) and Galiana-Merino et al. (2011) 110 have used the ratio of the semi-minor to the semi-major axis of the polarization ellipse to identify 111 Rayleigh waves contained in a seismic signal. This ratio is called in Galiana-Merino et al. (2011) 112 “instantaneous reciprocal ellipticity” (IRE), and is defined as:

113 where and are the semi-major and semi-minor axis of the ellipse, respectively. For a 114 three-component signal these axes are given by the following expressions (Pinnegar 2006): 


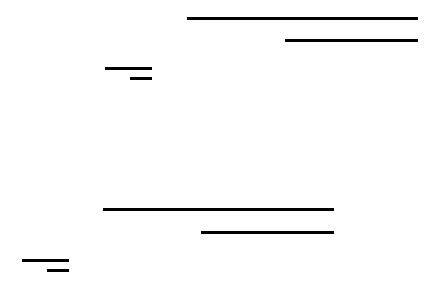

115 where

116 Here and are the real and imaginary parts of the Stockwell transform of the -component of the 117 signal (we omit the arguments to avoid clutter). Similarly, ( $\quad$ ) and $(\quad$ ) are pairs of the real 118 and imaginary parts of the Stockwell transform for the - and - components of the signal, respectively. 119 Note that the major axis of the polarization ellipse is composed of two parts, and . Since the 120 semi-minor axis corresponds to the radius of the circular polarization, the segment will be the 121 part corresponding to linear polarization. Therefore, if the polarization state is circular, , and 122 consequently If the polarization is linear, and then The IRE can then be 123 used to discern the different waves contained in a seismic signal. To identify Rayleigh waves, Galiana124 Merino et al. (2011) and Pinnegar (2006) have considered values of greater than . Using this 125 criterion, filters to extract the desired wave from the signal can be readily constructed. The filters are 126 applied in the time-frequency domain , and then the Inverse Stockwell Transform is used to 127 recover the filtered time-domain signal. The Inverse Stockwell Transform is computed in two steps: 
128 first, the Fourier Transform of the original signal is obtained by integrating the Stockwell transform over 129 time , and then the Fourier Transform is inverted (Stockwell et al., 1996).

130 Galiana-Merino et al. (2011) made use of the IRE to extract Rayleigh waves adopting the Stationary 131 Wavelet Packet Domain (SWPD) method. However their approach requires knowledge, in advance, of 132 the direction of propagation of the Rayleigh waves in order to be applied. In Galiana-Merino et al. 133 (2011) it is suggested that this direction will be the "radial" direction of wave propagation (i.e. the back134 azimuth to the epicenter). However this assumption may not be correct if the waves are generated by 135 diffraction at the edge of a basin, or if they are waves trapped inside the basin. Thus, in Galiana-Merino 136 et al. (2011) the horizontal components are rotated to obtain the radial component and then, the vertical 137 and radial components are used to compute the IRE. On the contrary, the IRE, defined by Pinnegar 138 (2006) as in Eq. (2), is computed using the three components of the signal and it does not change when 139 the horizontal components are rotated. However, even after extracting the waves (i.e. their components 140 along two arbitrary orthogonal axes), their direction of propagation is not provided by the filtering 141 process using the value of the IRE as the criterion.

\section{DIRECTION OF SURFACE WAVE POLARIZATION}

143 Once the polarization filtering has been completed, the angle of propagation of the extracted Rayleigh 144 waves may be estimated by correlating the filtered signals. One way to accomplish this is to use the 145 Chael-Selby-Baker-Stevens technique of calculating the back-azimuth of Rayleigh waves (Chael, 1997;

146 Selby, 2001; Baker and Stevens, 2004). The basic idea of this approach is to find an azimuth for which 147 the vertical and Hilbert-transformed radial component particle motions form a straight line (i.e. they are 148 linearly polarized). First, the two horizontal components are rotated into assumed radial and transverse 149 directions, with a trial back-azimuth ranging from to . The computed radial component is then 
150 shifted in time using the Hilbert Transform. The shift must be a phase delay for pro-grade motion or 151 phase advance for retro-grade motion (see Figure 1). The last step is the computation of the cross 152 correlation between the vertical and Hilbert-transformed horizontal (radial) traces with the following 153 formula:

154 where

$$
\text { , or }
$$

155 the function

is the vertical displacement component and

is the Hilbert-transformed radial

156 displacement component. The estimated direction of the Rayleigh wave propagation corresponds to the 157 direction ( ) that provides the maximum correlation, as sweeps the range to .

158 Clearly, the Chael-Selby-Baker-Stevens technique is a procedure that relies on sweeping the entire 159 parameter space and selecting the value that returns the highest correlation. Here we propose a more 160 direct procedure to compute the direction of propagation of the surface waves. Let us assume that the 161 recorded motion consists of three components: along the N-S direction (positive pointing to the 162 North); along E-W direction (positive pointing to the East); and in the vertical direction 163 (positive pointing up). Then, if the above-defined horizontal components were rotated by an angle 164 (positive clockwise, as shown in Figure 2), so as to render: the radial component (along the 165 direction of propagation; positive direction pointing away from the source/origin of the dispersive 166 wave); and the transverse component (in the transverse direction, obtained by rotating the radial 167 direction clockwise by ( 22 ), as shown in Figure 2). To derive an expression to directly compute , we 
169 follows:

170 If the radial component

171 phase, then we can reasonably consider that these are the components of a Rayleigh wave, which, in 172 turn, implies that, the correlation coefficient of the transverse component and should ideally 173 be zero (in essence we are assuming that the identified Rayleigh wave is not correlated with the linearly 174 polarized wave in the transverse direction, if such a wave exists). Under this assumption, Eq. (5) leads 175 to:

176 Substitution of the second equation given in (7) in the above expression leads to:

177 Solving this equation for we obtain the average direction of propagation of the wave train:

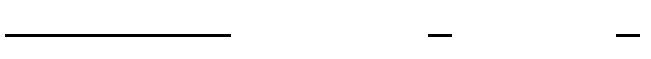

178 where the subscript is added because Eq. (10) provides only the 'reference' angle of the direction of 179 propagation of the Rayleigh waves. The azimuth in its correct quadrant can be computed with the 180 expressions: 
181 which can be condensed in the following single equation:

182 where is the sign function. Note that Eqs. (11) and (12) take into account the sign of reference 183 angle , which will have the same sign as . If the extracted signal is composed of more than one 184 dispersive wave propagating in distinct, albeit similar, directions, then, Eq. (10)-(12) should be applied 185 independently to each one of them. By inspecting the Stockwell Transform of the signal the analyst can 186 observe if there are several wave trains.

187 The time-domain procedures to compute the direction of propagation of Rayleigh waves presented in 188 this section work well if the waves have already been identified and extracted. An implicit assumption in 189 those procedures is that Rayleigh waves are either pro-grade or retro-grade, but not a mixture. Retro190 grade particle motion is usually the type of polarization expected for Rayleigh waves. However, some 191 geological settings allow for the generation of both retro-grade and pro-grade waves. One example is the 192 West Coastal Plain in Taiwan, as reported by Wang et al. (2006). With the IRE criterion as defined in 193 Eq. (2) it is not possible to identify whether the particle motion is pro-grade or retro-grade. Galiana194 Merino et al. (2011) suggest the instantaneous phase difference between the Radial and Vertical 195 components can be used to discern between these two types of motion. However, this requires 196 knowledge of the angle specifying the radial direction, and in most cases this angle is not available to 197 the analyst. This is why we propose a new criterion to filter the components of the signal to extract 198 Rayleigh waves, which does not require the specification of their direction of propagation, and 
199 differentiates between pro-grade and retro-grade motion. This criterion is the Normalized Inner Product 200 (NIP) that we define in the following section.

\section{THE NORMALIZED INNER PRODUCT}

202 Let the Stockwell Transforms of the North, East and Vertical components of the signal be denoted by 203 , , , respectively. In a similar manner, we denote the Stockwell Transforms of 204 the radial and transverse component of the signal by and . We recognize that each of 205 the discrete Stockwell Transforms is a matrix defined in the discretized space. Furthermore, each 206 element , of the discretized space is a complex number and may be expressed as 207 follows:

208 We find it convenient to treat each element as a two-element vector; e.g. for the vertical 209 component , we define:

210 with corresponding definitions for the radial, transverse, North and East components,

212 can define inner (dot) products with them. For example, the inner product of the radial with the vertical 213 component can be expressed as: 
214 The inner product allows one to take advantage of the following facts: 1) the phase of the

215 Stockwell Transform is absolutely referenced, (2) when normalized, the inner product is (in a way) the

216 time-frequency counterpart of the correlation in the time domain. Therefore, for a Rayleigh wave, if we

217 shift appropriately (i.e. by a phase-delay for pro-grade particle motion, or by phase-advance for retro-

218 grade particle motion) the vertical component, then the shifted vertical component should be in-phase

219 with the radial component. If we refer to the shifted vertical component by , then ideally

222 values close to 1. Making use of the definitions established earlier, the Normalized Inner Product of the 223 radial and appropriately shifted vertical components, denoted by is given by:

224 Note that in the time-frequency domain, the time shifted vertical component is obtained simply 225 by multiplying the positive frequencies of $\quad$ by for a phase advance, and by 226 - for a phase delay. Then, we can construct simple filters to retain only those regions in the 227 space where the value of the $\quad$ is close to 1 (say, and setting the rest of the 228 space equal to zero. Following Pinnegar (2006), the filters can be alternatively defined using 229 continuous functions by means of cosine tapers, to reduce numerical artifacts when the filtered 230 transforms are inverted to recover the extracted waves.

231 Since we do not know the direction of propagation of the Rayleigh wave, the elements cannot 232 be computed directly applying the Stockwell Transform to some time history in such direction. Here we 
233 propose an indirect method to compute the elements

. Exploiting the linearity of the Stockwell

234 Transform, we obtain the time-frequency counterpart of Eq. (7):

235 In order to find the azimuth , we again make use of the fact that, for a Rayleigh wave, the correlation

236 between the transverse component and the shifted vertical component is zero:

237 Following a similar reasoning to the one presented in the previous section, the time-frequency 238 counterpart of Eq. (10) can be expressed as:

239 Here is a function of $\quad$, and is expected to present small variations when associated to a wave 240 train. If several wave trains are present in the signal, having different directions of propagation, Eq. (19) 241 is valid for each of the corresponding time intervals. Now, taking in consideration the quadrants in the 242 N-S, E-W plane, the azimuth giving the direction of propagation of the wave train is given by:

243 where 
246 the sense of propagation can be accomplished if the position of the source/origin of the signal is known, 247 or if we have more than one station recording the propagating dispersive wave. Let us emphatically note 248 that if the sense of propagation, of the phase under investigation, is not established, pro-grade or retro249 grade motion cannot be defined without ambiguity. Also, let us note that sense of propagation and 250 direction of propagation are not the same thing. The direction is given by a numerical value of , 251 whereas the sense of propagation only indicates whether the propagation of the wave train is towards the 252 East or the West. Once the angle is computed, the elements can be computed in the 253 time-frequency domain with the first equation given in (17).

254 The NIP criterion is particularly useful when in the seismogram we have simultaneously the traces of 255 pro-grade and retro-grade Rayleigh waves. These waves may be associated with different frequencies if 256 the physical processes that generate them are different. Since the NIP criterion we use is defined in 257 terms of the filter constructed with this criterion will exclude the regions of the space 258 that are associated with pro-grade particle motion if has been obtained with a phase advance. 259 Conversely, the filter will exclude the regions of the space corresponding to retro-grade motion if 260 has been obtained with a phase delay (Figure 1).

261 After the filters are applied to the time-frequency components of the signal, the filtered Stockwell 262 Transforms are inverted and what we eventually obtain are Rayleigh waves with only pro-grade or retro263 grade particle motion (depending on how we shifted the vertical component). The radial, transverse and 264 vertical components can then be obtained by either of the following two approaches: 
1) Applying the filter to the , and components, and inverting the resulting

266 Transforms to obtain the North-East-Vertical components of the extracted wave train. Then, the radial267 transverse components are obtained by rotation of the North-East components with the azimuth 268 calculated with Eq. (12).

\section{Extraction of Love Waves}

273 The criterion is also useful for the extraction of Love waves, which are dispersive waves 274 linearly polarized on the horizontal plane along a direction which is transverse to the direction of 275 propagation. In the case of a noise-free synthetic signal which consists of only linearly polarized waves 276 on the horizontal plane, would be zero and apparently Eq. (19) could not be used. However if 277 we express Eq. (19) in the following manner:

278 which can be simplified as:

279 Thus, the computation of is not affected by the zero amplitude of . If we consider also 
281 Therefore, in this case is going to provide the direction of polarization of the Love wave, not its

282 direction of propagation. The wave trace is going to be found in the radial component computed 283 with Eq. (17). Now, in the case of real data we can reasonably argue that if for no other 284 reason but for the presence of ambient noise. If in fact this is the case, then Eq.(18) can be used and, 285 along with Eqs.(19) \& (20), provides an estimate of the direction of propagation of the Love wave. In 286 this case the wave trace is going to be found in the transverse component

\section{WAVE EXTRACTION USING SYNTHETIC SIGNALS}

288 In this section, we illustrate the application of our proposed procedure to extract waves from synthetic 289 signals. We consider an example very similar to the one presented by Galiana-Merino et al. (2011), 290 where a constructed synthetic signal is used. To construct the synthetic signal we combine three 291 windowed sinusoids with frequencies: 5, 2 and $1 \mathrm{~Hz}$, shown in Figure 3, and denoted by , 292 and , respectively. In order to include elliptically polarized waves, we apply a phase advance of /2 293 rad to the signal _ and a phase delay of $/ 2 \mathrm{rad}$ to the signal _. The resulting signals are denoted 294 by and , respectively. The three frequencies are then combined as shown in shown in Figure 295 4, to obtain a three-component signal, which is simultaneously linearly and elliptically polarized. As 296 Figure 4 indicates, we choose the pro-grade wave of $1 \mathrm{~Hz}$ to be in the - plane, with following 297 components: in the -direction, and in the -direction. On the other hand, the retrograde 298 wave of $2 \mathrm{~Hz}$ is assigned to the - plane, with the following components: in the -direction 
300 defined as - $\quad$ in both - and - directions. Since both the and components have the same

301 amplitude, the linearly polarized wave has a direction of propagation of 45 degrees measured clockwise 302 from the positive -axis.

303 Now, we translate the - - coordinate system into a North-East-Vertical system. For this, we assign an 304 azimuth of 60 degrees (measured clockwise from North) to the retro-grade wave (which propagates 305 along the -axis). Using a right-handed coordinate system, the direction of the other two waves will then 306 be as follows: the azimuth of the pro-grade wave (which propagates along the -axis) will be 150 $307(=60+90)$ degrees, and the azimuth of the wave linearly polarized in the $x-y$ plane will be $105(=60+45)$ 308 degrees. We then rotate the $x$ and $y$ components to obtain the North-East components by means of the 309 rotation matrix:

310 The resulting $N-E-V$ components are shown in Figure 5. We can observe the three frequencies are 311 superimposed and there is no visual indication of the type of waves contained in the signal. We will 312 work with these components as starting data for our proposed procedures, since most available 313 seismograms are given in $N-E-V$ components.

\section{Extraction of Retro-grade Rayleigh wave}

316 In this section we will apply filtering to extract the retro-grade wave, that is, the 2-Hz elliptically 317 polarized wave in the $y$-z plane. We will use both, the IRE, and independently, the NIP, as criteria to 
construct and compare the filters. Once the wave is extracted we will recover the azimuth that was

319 assigned to the retro-grade wave in the previous section. We start by computing the Stockwell 320 Transform of the - $-V$ components, whose amplitudes are shown in Figures 6(a)-(c). Using the 321 notation introduced in the previous section, , and , shown in Figure 6(d). Because of the color scale distributed in using , each frequency is rotated according to its corresponding is derived under the assumption that the correlation between the transverse and observe that there is no energy in the transverse component for all frequencies. This is an expected result because the angle ero. It is important to clarify that when we compute the component vertical components is zero. It is important to clarify that when we compute the , we use equation (17) to component" in the full time-frequency space will not have the physical meaning of the Stockwell Transform of a component of the signal in a specific "radial” direction. However, such computation is useful for extracting waves, since after we apply filtering, only one wave (or wave train) will remain in the time-frequency domain, and with it, only one direction of propagation. The next step is the computation of NIP , obtained by applying equation (16) and shown in Figure 7(a). Since we want 
341 to extract the wave with retro-grade motion, we set

342 corresponds to the presence of the retro-grade wave (the NIP
. The red region in Figure 7(a)

), whereas the black region

343 corresponds to the pro-grade wave (the NIP ). For the linearly polarized wave, the values of

344 NIP oscillate around zero. This unstable behavior [not shown in figure 7(a)] is due to the presence

345 of the amplitude of in the denominator in Eq. (16); the amplitude of is zero for a wave

346 linearly polarized in the - plane. This situation, however, is unlikely to be found in real seismograms

347 because the presence of noise will keep the amplitude of different from zero. Then, in order to

348 avoid this unstable behavior when dealing with noise-free synthetic signals, the amplitude used

349 in Eq. (16) may be modified in the following manner:

350 where is the tolerance for small values of

. Because of the finite energy

351 carried by seismic waves, we expect

to be bounded. In Figure (7a) the

is computed

352 adopting a tolerance $\quad$. Now, for comparison, the IRE is also shown in Figure 7(b), which was

353 obtained by applying equations (2)-(4). We can observe that the regions corresponding to both the pro-

354 grade and retro-grade wave have an IRE of about 0.5. As expected, the IRE is zero in the region of the 355 linearly polarized wave.

356 Now that the waves have been identified, we construct filters to isolate or extract the elliptically 357 polarized (retro-grade) wave. The continuous filters based on the NIP and the IRE are constructed 358 as follows: 
359 where is the threshold value, and the width of the cosine taper. In this example we selected, $360 \quad$, and to construct the filter using as criterion the value of the IRE. On

361 the other hand, for the filter using as criterion the value of the NIP, we selected

$362 \quad$, and Since we already computed the component _ the filters can be applied to it, 363 and to the vertical component to extract directly the Rayleigh waves. In Figures 7(c)-(d) we 364 compare the filtered component using the NIP criterion [Figure 7(c)], and the IRE 365 [Figure 7(d)]. It is evident that filtering with the IRE criterion alone does not separate the pro-grade and 366 retro-grade waves in this example. On the other hand, Figure 7(c) shows the effectiveness of the 367 NIP criterion to isolate the retro-grade (Rayleigh) waves present in the synthetic signals.

368 Finally, the desired wave is extracted by computing the inverse Stockwell transforms after the and $\quad$ components are filtered with the NIP criterion. Figure 8 shows the radial, 370 transverse and vertical components of the unfiltered signal and those of the extracted wave in the time 371 domain. In the previous section we selected the $y$-axis as the direction of propagation of the retro-grade 372 wave, and thus, the $y$-axis is the "radial" direction for this wave. As expected, there is no extracted wave 373 in the transverse direction (the pro-grade wave which would have appeared on the transverse component 374 has been eliminated by filtering).

375 Let us note that we use to compute the NIP , and then we use the NIP to filter and 376 extract the desired wave. However, even though the components of the extracted wave are already 377 obtained in the radial and transverse direction, the unique numerical value giving the azimuth of such 
378 directions has not been provided. If such azimuth is also desired, it can be computed by filtering the 379 and components with the NIP

criterion. Then, the angle is computed using Eqs. (10)-(12) once the time-domain $N-E-V$ components are obtained inverting the filtered and components. The $N-E-V$ components of the extracted retro-grade wave computed in this manner are shown in Figure 9. The extracted wave has a frequency of $2 \mathrm{~Hz}$, as expected. Now, applying Eqs. (10)-(12) to these $N-E-V$ components, we obtain an azimuth for the retrograde Rayleigh wave of 59.9994 degrees, the expected value. Let us note that the $R-T-V$ components of Figures 8(d)-(f) were obtained by rotating the $N-E-V$ components of Figure 5 with the computed azimuth.

\section{Extraction of Pro-grade Rayleigh Wave}

To extract the pro-grade Rayleigh wave from the synthetic signal we follow the same procedure as in the previous section, but now we set , for a phase delay for the vertical component

have been interchanged [compare Figure 10(a) with Figure 7(a)], since now we are targeting the wave of $1 \mathrm{~Hz}$ (which, we remind the reader, is the pro-grade wave propagating along the $x$-axis, that is, an azimuth of 150 degrees). When we apply the filter, based on the NIP criterion, to the component, we can observe that the only remaining wave has a frequency of about $1 \mathrm{~Hz}$, as shown in Figure 10(b). Finally, the radial, transverse and vertical component of the unfiltered signal and extracted wave are shown in Figure 11. Once again, there is no component in the "transverse" direction of the extracted wave. The azimuth giving the direction of propagation of the pro-grade wave, obtained by applying Eqs. (10)-(12) is 150.0011 degrees. As expected, in this case the "radial" direction coincides with the $x$-axis of the coordinate system, as shown in Figure 11. 
400 To verify that we in fact have extracted retro-grade and pro-grade Rayleigh waves, we inspect their 401 polarization characteristics. In Figure (12) we compare the radial and shifted vertical components of the 402 extracted waves. For the retro-grade, the vertical component is shifted with a phase advance, and 403 for the pro-grade wave, with a phase delay. Since the compared components are clearly in-phase, 404 we can conclude that we have extracted Rayleigh waves.

\section{Extraction of Linearly Polarized wave}

406 Now we consider the problem of extracting the wave that is linearly polarized in the - plane. In such a 407 case, Love waves and shear waves would be the candidates. For Love waves we would expect the 408 presence of a dispersed wave train. Note that to extract the linearly polarized wave, the component can 409 be obtained either way, by shifting with a phase advance, or a phase delay. In Figure (13a) the was computed shifting with a phase delay. We can observe that the values of the

411 for the $5 \mathrm{~Hz}$ wave are close to zero, a result consistent with linear polarization. Thus, in this case the

413 follows: component is the wave of $5 \mathrm{~Hz}$. Next, the radial, transverse and vertical components of the

417 unfiltered signal and extracted wave are shown in Figure (14). Here the $y-x$ components of the unfiltered 
418 signal have been rotated 45 degrees, to obtain its radial and transverse components. Even before filtering 419 we can already observe that the high frequency wave is present only in the "radial component. 420 Regarding the extracted wave, Figure (14) shoes that only the radial component of the extracted signal is 421 non-zero, consistent with what it was anticipated. Now, in this case of a noise-free synthetic signal, Eq. 422 (10) is not appropriate to compute the azimuth of the direction of polarization of the wave, since the 423 time-domain component is zero. However, we can take advantage of the fact that we have already 424 extracted the radial component of the wave. Let us note that for a wave linearly polarized on the 425 horizontal plane:

426 if is in the direction of polarization. Thus, a new expression can be obtained, in the same manner 427 we derived Eq. (10):

428 After computing the North and East components of the extracted wave (inverting the filtered 429 and components) the azimuth obtained using Eqs. (30) and (12) is 105.0004. It is important to 430 remark that this azimuth gives the direction of polarization. If the extracted wave is a Love wave, its 431 direction of propagation would be perpendicular to it.

\section{AN EXAMPLE WITH REAL SIGNALS}

433 In this section we apply the procedure for identification and extraction of surface waves produced by an 434 aftershock of the Chi-Chi earthquake in the West Coastal Plain (WCP) in Taiwan, which occurred on 
435 September, 20th 1999 at 1803 UTC with a magnitude of Mw 6.2. This aftershock is very useful to test

436 the proposed method to extract surface waves, since it produced very strong and clear surface waves in 437 the WCP. Besides, since Rayleigh and Love waves had been previously identified from the recordings 438 and reported in the literature (e.g., Wang et al., 2006) this data set allows us to assess with confidence if 439 we are in fact extracting Rayleigh and Love waves. Figure (15) shows a map with the directions of the 440 Rayleigh wave propagation obtained by Wang et al. (2006), where the location of the epicenter is also 441 indicated. The circle in Figure (15) specifies the location of station TCU116, which we will consider for 442 this example.

\section{Extraction of Retro-grade Rayleigh waves}

444 The $N-E-V$ components of the displacement time history at station TCU116 during the aftershock are 445 shown in Figure (16). The displacements were obtained by independently bandpass filtering between 0.1 446 and $10 \mathrm{~Hz}$ and integrating twice the components of the acceleration histories. We can observe from the 447 time histories in Figure (16) that most of the energy of the signal is contained in the East and Vertical 448 components. The Stockwell Transforms of the displacements histories are computed, and with them, we 449 compute the angle using Eqs. (19)-(20). Then, the components and are 450 computed according to Eq. (17). The results are shown in Figure (17). In Figure 17(a) we can observe 451 regions in the time-frequency domain (between 20 and 50 seconds) with small variations of the values of 452 , which can be associated to wave trains. We find very little energy in the component, 453 even in this case of a real signal, since is derived under the assumption of zero correlation 454 between the and components. The next step is the computation of NIP, obtained 455 by applying equation (16) and shown in Figure 18(a). Since we want to extract Rayleigh waves with 456 retro-grade motion first, 
458 The region corresponding to the retro-grade motion is indicated by the red color. For comparison, the 459 IRE is also shown in Figure 18(b). We can conclude from the comparison that the NIP is more 460 stable over the domain, as opposed to the IRE, which has more variation in the Stockwell 461 transform domain. In Galiana-Merino et al. (2011), it is even stated that a 2D filter needs to be applied 462 to the IRE obtained with the SWPD, because its high variation could lead to numerical problems. In this 463 example, since we are not dealing with synthetic signals, the NIP is computed with Eq. (16) $464 \quad$ without making any modification to _ and without any smoothing.

$465 \quad$ Figure 18(c) shows the component filtered with the NIP criterion, according to Eq. (27). 466 The same component filtered with the IRE criterion is shown in Figure 18(d). The results of this 467 example clearly demonstrate that filtering with the NIP criterion effectively isolates the retro468 grade wave from the pro-grade wave. Upon inverting the filtered transforms, we obtain the extracted 469 retro-grade wave. In Figure (19) we can observe the radial, transverse and vertical components of the 470 unfiltered signal and the extracted retro-grade wave. The comparison between the unfiltered signal and 471 extracted wave shows that filtering has excluded a wave component observed between 25 and 35 472 seconds. Such component would not have been excluded filtering with the IRE criterion alone. We can 473 also observe that the transverse component of the extracted wave is minimized. The correlation 474 coefficient computed as in Eq. (5) between the radial and shifted (with a phase advance) vertical 475 components of the extracted wave is 0.91097, showing in a quantitative manner the good results that are 476 obtained when the NIP criterion is used. Finally, the azimuth of the direction of propagation of the 477 Rayleigh wave is computed with Eqs. (10)-(12), obtaining a value of 257.9926 degrees. This result is 478 compatible with the directions reported by Wang et al. (2006), indicated in Figure (15). The angle of 
479257.9926 degrees was used to rotate the $N-E-V$ components of the unfiltered signal to obtain the 480 components $R-T-V$ components shown in Figures 19(a)-(c).

481 Extraction of Pro-grade Rayleigh waves

482 Figure 20(a) shows NIP to extract the pro-grade Rayleigh waves, and Figure 20(b) shows the 483 component when filtered with this criterion. We observe that the pro-grade wave train has 484 different frequency content (higher frequencies) than that of the retro-grade wave train, and that it is 485 located at around 30 seconds. Figure 21 shows the radial, transverse, and vertical components of the 486 unfiltered signal and extracted pro-grade wave. We can observe the different time location of the pro487 grade wave relative to the previously extracted retro-grade wave. With the use of the NIP criterion, we 488 have managed to separate these waves, even in the time range in which they overlap. Certainly, such 489 separation is possible because of the different frequency content of the waves. The correlation 490 coefficient of the radial and shifted (with a phase delay) vertical components of the extracted pro-grade 491 wave is 0.91034 . The computed azimuth is 282.6976 degrees. This azimuth is significantly different 492 from the one obtained for the retro-grade waves (257.9926 degrees), indicating that probably the 493 observed pro-grade and retro-grade Rayleigh waves are generated in different manners.

494 Finally Figure (22) shows the comparison between the radial and shifted vertical components of the 495 extracted Rayleigh waves. Clearly, the radial component and the vertical component shifted with a 496 phase advance shown in Figure 22(a) are in phase, confirming the extracted waves of Figure 22(a) are 497 retro-grade Rayleigh waves. In Figure 22(b) the vertical component is shifted with a phase delay, 498 confirming that the extracted wave shown in Figure 22(b) is a pro-grade Rayleigh wave. 
500 In Figure (17) we can observe that the component at station TCU116 does not show the

501 presence of a wave train that can be associated to Love waves. This is why we analyze the recording of 502 the same event at station TCU118, for which Wang et al. (2006) had already identified Love waves. For 503 this station we first extract the retro-grade Rayleigh wave train, following the procedure detailed in the 504 previous section. The components of the extracted retro-grade Rayleigh wave are shown in Figure 23(a). 505 Let us note that the resulting azimuth giving the direction of propagation of the retro-grade Rayleigh 506 wave train is 304.51 degrees, a result close to the 297 degrees reported in Wang et al. (2006). With this 507 direction we use Eq. (7) to compute the radial and transverse components of the unfiltered signal in the 508 time domain, and then compute their Stockwell Transforms, which are shown in Figure (24). A wave 509 train with a later arrival time (relative to the Rayleigh wave's arrival) is clearly present in the time510 frequency transverse component of the unfiltered signal, as shown in Figure 24(b). The Love waves are 511 effectively extracted using the filter based on the criterion defined in Eq. (27). Even though 512 we could use the criterion and construct a filter according to Eq. (28), the filter given in Eq. 513 (23) is more convenient because the is more stable in the space. The extracted Love 514 waves are shown in Figure 23(b). The waveforms and corresponding arrival times of the waves 515 extracted with our proposed procedure are similar to those reported in Figure 9 of Wang et al., (2006).

516 Now, to illustrate the performance of our procedure to extract surface waves in high noise conditions, 517 we analyze the recording at station CHY107. This station is located farther from the epicenter, to the 518 south of the WCP, as shown in Figure 15. The strength of the wavefield at such far location is weak. A 519 comparison of the unfiltered signal and the results of applying filtering using the NIP criterion are 520 shown in Figure 25. We can observe that much of the noise has been filtered out when the NIP criterion 521 is used. The correlation coefficient between the radial and shifted vertical components is 0.8449, giving 
522 a strong indication that the extracted (retro-grade) wave in the radial direction is a Rayleigh wave. This 523 high correlation is illustrated in Figure 25(d), which shows the comparison of the radial and phase 524 advanced vertical component. Furthermore, the azimuth obtained with the extracted waves is 226.22 525 degrees, a direction consistent with the location of station CHY107 relative to the epicenter, shown in 526 Figure 15. We can also observe a strong intensity (relative to the radial component) in the transverse 527 component of the extracted waves. Even though we could consider the extracted waves in this transverse 528 component as Love waves, the extracted signal might as well be simply linearly polarized noise. In cases 529 like this, with a relatively lower signal-to-noise ratio, better results can be obtained if the signal is 530 denoised before applying filtering to extract Love waves.

531 Finally, we have applied the NIP criterion to extract Rayleigh and Love waves to other stations at the

532 WCP in Taiwan. Figure 26 illustrates the extracted retro-grade and pro-grade Rayleigh waves at each 533 station. The Love waves, extracted from the transverse component of the retro-grade Rayleigh waves are 534 shown in Figure 27. The results show the extraction method is stable when applied to this dataset of 535 seismic time histories. The computed azimuths of retro-grade Rayleigh wave propagation are in 536 excellent agreement with those obtained by Wang et al. (2006), indicated in Figure (15).

537 CONCLUSIONS

538 We have proposed and developed a method to extract Rayleigh and Love waves from three-component 539 displacement histories. The proposed method, based on the Normalized Inner Product (NIP), does not 540 require a priori estimations of the frequency range and direction of propagation of the surface waves. 541 We have shown that the method proposed herein distinguishes between pro-grade and retro-grade 542 particle motion. Therefore surface waves with such different polarization characteristics can be more 543 easily identified. Furthermore, examples with real signals show that the Normalized Inner Product is 
544 more stable over the time-frequency domain than the Instantaneous Reciprocal Ellipticity. We also 545 showed that the proposed method works well for extracting Love waves from noise-free synthetic 546 seismograms as well as from real seismograms. The method was applied to extract Rayleigh and Love 547 waves from displacement histories of aftershock 1803 of the Chi-Chi earthquake recorded at the 548 Western Coastal Plain in Taiwan. The extracted waves and corresponding directions of propagation are 549 in excellent agreement with previous surface wave analysis reported in the literature.

\section{DATA AND RESOURCES}

551 All recorded seismograms used in this work are from CD-002 titled "CWB Free-Field Strong-Motion 552 Data from Three Major Aftershocks of the 1999 Chi-Chi Earthquake: Processed Acceleration Data Files 553 on CD-ROM" prepared in 2001 by W. H. K. Lee, T. C. Shin, and C. F. Wu of the Seismological 554 Observation Center, Central Weather Bureau, Taiwan.

555 The relief geographic map of the West Coastal Plain was generated with the code READHGT written by

556 François Beauducel, from the Institute de Physique du Globe de Paris. Input data for the Digital 557 Elevation Map was downloaded from http://dds.cr.usgs.gov/srtm/version2_1. Coast lines were extracted 558 from http://www.ngdc.noaa.gov/mgg/coast/getcoast.html. Both websites were last accessed in January 5592014. 
561 The authors are grateful to two anonymous reviewers for constructive comments which improved the 562 clarity of the manuscript. The authors are also indebted to Dr. Fabian Bonilla for fruitful discussions on 563 surface wave propagation, and for introducing us to the use of the Stockwell transform for analysis of 564 non-stationary signals.

565 This research has been co-financed by Electricité de France (EDF) through the MARS project and by the 566 European Union (European Social Fund - ESF) and Greek national funds through the Operational 567 Program "Education and Lifelong Learning" of the National Strategic Reference Framework (NSRF) 568 Research Funding Program: THALES. Investing in knowledge society through the European Social 569 Fund.

\section{REFERENCES}

571 Baker, G.E. and Stevens, J.L. (2004). "Backazimuth estimation reliability using surface wave 572

573 Chael, E.P. (1997) “An automated Rayleigh-wave detection algorithm”, Bull. Seism. Soc. Am. 87, 157574 163.

Dziewonski, A., Bloch, S. and Landisman, M. (1969) "A Technique for the Analysis of Transient Seismic Signals”, Bull. Seismol. Soc. Am., 59(1), 427-444. polarization”, Geophysical Research Letters, 31, L09611, doi:10.1029/2004GL019510. 
579 Galiana-Merino J.J., Parolai, S., and Rosa-Herranz, J. (2011). "Seismic wave characterization using 580 complex trace analysis in the stationary wavelet packet domain”, Soil Dynamics and Earthuqake $581 \quad$ Engineering. 31(11), 1565-1578.

582 Li, X.L., Crampin S. (1991) “Complex component analysis of shear wave splitting: theory”, 583 Geophysical Journal International. 107, 597-604.

584 Pinnegar, C.R. (2006). "Polarization Analysis and polarization filtering of three-component signals with 585 the time-frequency S transform”, Geophysical Journal International. 165, 596-606.

586 René, R.M., Fitter, J.L., Forsyth, P.M., Kim K.Y., Murray, D.J., Walters, J.K. (1986) “Multicomponent 587 seismic studies using complex trace analysis”, Geophysics. 51, 1235-1251.

588 Selby, N.D. (2001) “Association of Rayleigh waves using back-azimuth measurements: application to 589 Test Ban verification”, Bull. Seism. Soc. Am. 91, 580-593.

590 Smart, E. (1978) “A Three-Component, Single-Station, Maximum-Likelihood Surface Wave Processor”, 591 Seismic Data Analysis Center Report, SDAC-TR-77-14, 62 pp.

592 Stockwell, R.G. (2007) "Why use the S-transform” in Pseudo-Differential Operators: PDEs and Time593 Frequency Analysis. (Fields Institute Communications), 52, pp. 279-309.

594 Stockwell, R., Mansinha, L., Lowe, R. (1996) "Localization of the Complex Spectrum: The S 595 Transform”, IEEE Transactions on Signal Processing. 44 (4), 998-1001.

596 Vidale, J.E., (1986) “Complex polarization analysis of ground motion”, Bull. Seismol. Soc. Am., 76(5), 597 1393-1405. 
598 Wang, G., Tang, G., Boore, D., Burbach, G., Jackson, C., Zhou, X., and Lin, Q. (2006). “Surface Waves 599 in the Western Taiwan Coastal Plain from an Aftershock of the 1999 Chi-Chi, Taiwan, 600 Earthquake”, Bull. Seism. Soc. Am. 96, 821-845.

601 AUTHORS' AFFILIATIONS:

602 (1) Universidad Nacional Autónoma de Honduras, Tegucigalpa, Honduras. E-mail: 603 kristelmeza@unah.edu.hn

604 (2) Department of Civil Engineering, University of Patras, GR-26500 Patras, Greece. E-mail: 605 papaga@upatras.gr

606 (3) Université Paris-Est, IFSTTAR, Départ. GERS, 20 Boulevard Newton, Champs sur Marne, France. 607 E-mail: jean-francois.semblat@ifsttar.fr.

608 FIGURE CAPTIONS

609 Figure 1. Time shift for vertical component. (a) For pro-grade motion (phase delay) (b) For retro-grade 610 motion (phase advance).

611 Figure 2. Reference system for direction of propagation of Rayleigh waves.

612 Figure 3. Windowed sinusoids to construct synthetic signal. (a) $\quad$ : $5 \mathrm{~Hz}$, (b) $\quad$ : $1 \mathrm{~Hz}$, (c) $\quad$ :2 Hz.

613 Figure 4. Three-component synthetic signal. (a) 
615 Figure 5. Three-component synthetic signal. (a) North component, (b) East component, (c) Vertical 616 component.

617 Figure 6. Time-frequency representation of synthetic signal. (a) Amplitude of North component, (b) 618 Amplitude of East component, (c) Amplitude of vertical component, (d) Azimuth of direction of 619 propagation according to Eq. (20), (e) Amplitude of radial component (f) Amplitude of transverse 620 component.

621 Figure 7. Comparison of filtering using the IRE and NIP criteria. (a) Normalized inner product of radial 622 and phase advanced vertical component, (b) IRE computed with the three-components of the signal, (c) 623 Radial displacement component filtered using the NIP criterion, (d) Radial displacement 624 component filtered using the IRE criterion.

625 Figure 8. Rayleigh retro-grade wave extracted from synthetic signal. (a) Unfiltered transverse ( -) 626 component, (b) Unfiltered radial ( -) component, (c) Unfiltered ( -) vertical component, (d) Extracted 627 transverse component, (e) Extracted radial component, (f) Extracted vertical component.

628 Figure 9. Extracted retro-grade Rayleigh wave. (a) North component, (b) East component, (c) Vertical 629 component.

630 Figure 10. Extraction of pro-grade wave using the NIP criterion. (a) Normalized inner product of radial 631 and phase delayed vertical component, (b) Amplitude of component filtered with the NIP 632 criterion. 
633 Figure 11. Rayleigh pro-grade wave extracted from synthetic signal. (a) Unfiltered radial ( -) 634 component, (b) Unfiltered transverse ( -) component, (c) Unfiltered ( -) vertical component, (d) 635 Extracted radial component, (e) Extracted transverse component, (f) Extracted vertical component.

636 Figure 12. Comparison of radial and shifted vertical components of extracted waves. (a) Retro-grade 637 wave (2 Hz), (b) Pro-grade wave (1 Hz).

638 Figure 13. Extraction of linearly polarized wave using the NIP criterion. (a) Normalized inner product of 639 radial and phase delayed vertical component, (b) Amplitude of component filtered with the NIP 640 criterion.

641 Figure 14. Linearly polarized wave extracted from synthetic signal. (a) Unfiltered radial component, (b) 642 Unfiltered transverse component, (c) Unfiltered vertical component, (d) Extracted radial component, (e) 643 Extracted transverse component, (f) Extracted vertical component.

644 Figure 15. Map illustrating the location of the stations on the West Coastal Plain considered in this 645 study. For some stations the arrows indicate the direction Rayleigh wave propagation estimated by 646 Wang et al. (2006). The star indicates the location of the epicenter of the event. The black circle 647 indicates the location of station TCU116.

648 Figure 16. $N-E-V$ components of the displacement history (in $\mathrm{cm}$ ) for Chi-Chi aftershock 1803 at station 649 TCU116. (a) North component (b) East component (c) Vertical component.

650 Figure 17. Radial and Transverse displacement components for the NIP at station TCU116. (a) Azimuth 651 of direction of propagation, (b) Amplitude of component (c) Amplitude of component. 
652 Figure 18. Comparison of filtering to extract the retro-grade wave from recording at station TCU116 653 using the IRE and NIP criteria. (a) Normalized inner product of and phase advanced

654 component, (b) IRE computed with the three-components of the signal, (c) component filtered 655 with the NIP criterion, (d) component filtered with the IRE criterion.

656 Figure 19. Rayleigh retro-grade wave extracted at station TCU116. (a) Unfiltered radial component, (b) 657 Unfiltered transverse component, (c) Unfiltered vertical component, (d) Extracted radial component, (e) 658 Extracted transverse component, (f) Extracted vertical component.

659 Figure 20. Extraction of pro-grade Rayleigh wave from recording at station TCU116. (a) Normalized 660 inner product of and phase delayed component, (b) Amplitude of component 661 filtered with the NIP criterion.

662 Figure 21. Rayleigh pro-grade wave extracted at station TCU116. (a) Unfiltered radial component, (b) 663 Unfiltered transverse component, (c) Unfiltered vertical component, (d) Extracted radial component, (e) 664 Extracted transverse component, (f) Extracted vertical component.

665 Figure 22. Comparison of radial and shifted vertical components of extracted Rayleigh waves at station 666 TCU116. (a) Retro-grade wave, (b) Pro-grade wave.

667 Figure 23. $R-T-V$ displacement components of extracted waves at station TCU118. (a) Comparison of 668 radial and shifted vertical component of retro-grade Rayleigh wave (b) Extracted (Love) wave in 669 transverse direction.

670 Figure 24. Radial and Transverse time-frequency components for unfiltered recording at station 671 TCU118. (a) Amplitude of radial component (b) Amplitude of transverse component. 
672 Figure 25. Extracted waves from recording at station CHY107. (a) Unfiltered radial component, (b) 673 Unfiltered transverse component, (c) Unfiltered vertical component, (d) Extracted radial component 674 (black line) and shifted vertical component (gray line), (e) Extracted transverse component, (f) Extracted 675 vertical component.

676 Figure 26. Radial component (black solid line) and shifted vertical component (gray dashed line) of 677 extracted Rayleigh waves at different stations of the WCP plain in Taiwan. (a) Retro-grade waves, (b) 678 Pro-grade waves.

679 Figure 27. Extracted Love waves at different stations of the WCP plain in Taiwan. 


\section{FIGURES}

(a)

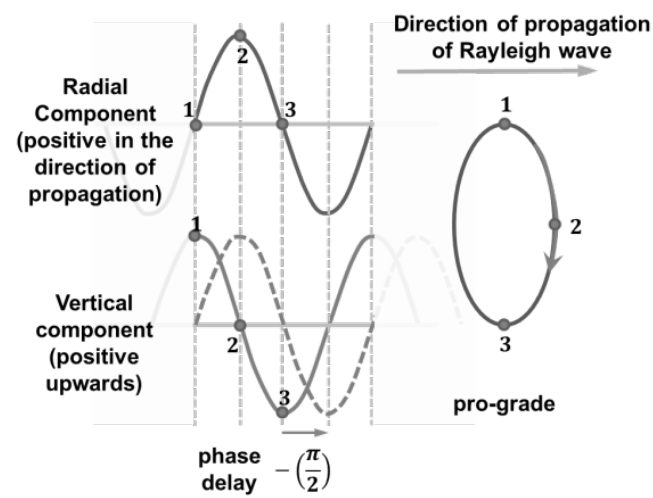

(b)

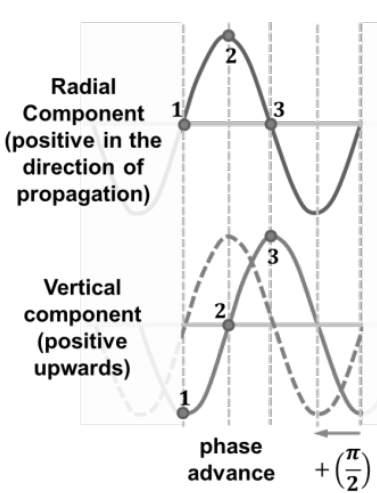

Direction of propagation of Rayleigh wave

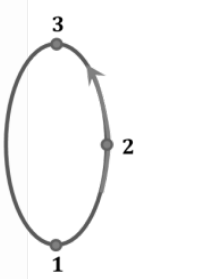

retro-grade

Figure 1. Time shift for vertical component. (a) For pro-grade motion (phase delay) (b) For retro-grade motion (phase advance)

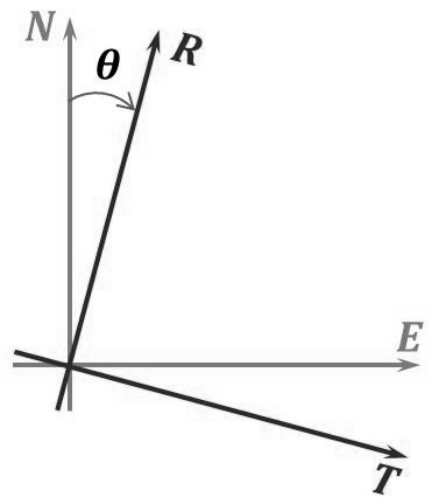

Figure 2. Reference system for direction of propagation of Rayleigh waves.

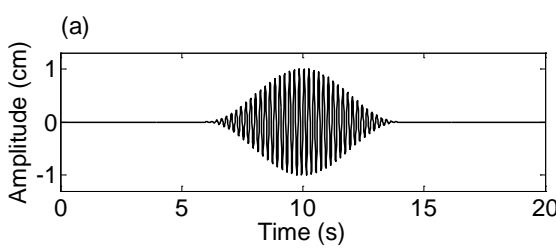

(b)

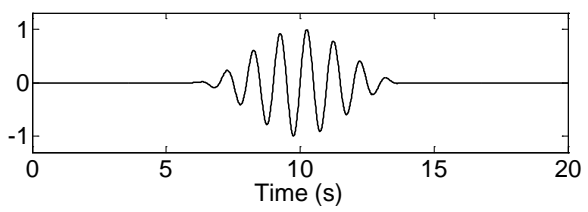

(c)

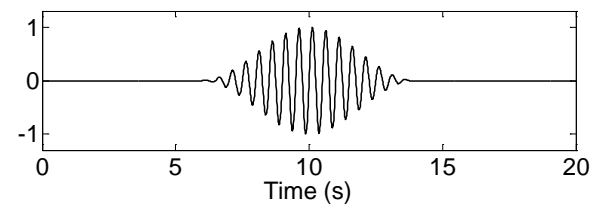

Figure 3. Windowed sinusoids to construct synthetic signal. (a) $\quad: 5 \mathrm{~Hz}$, (b) $\quad: 1 \mathrm{~Hz}$, (c) $\quad: 2 \mathrm{~Hz}$.

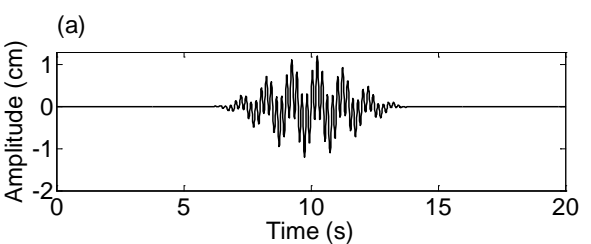

Figure 4. Three-component synthetic signal. (a) (b)

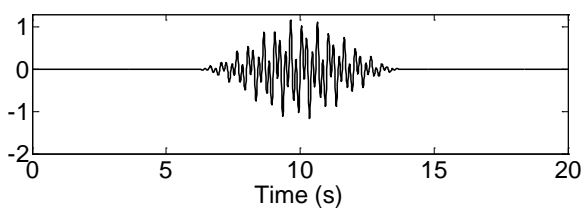

$-$ (c)

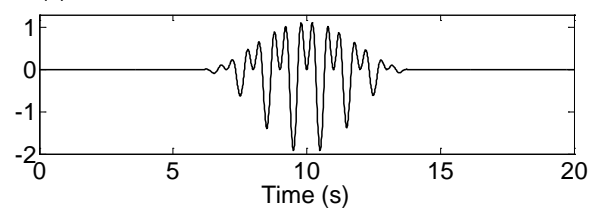

, (b)

(c) 
(a)

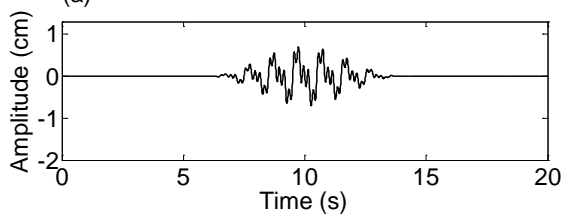

Figure 5. Three-component synthetic signal. (a) North component, (b) East component, (c) Vertical component.

(a)

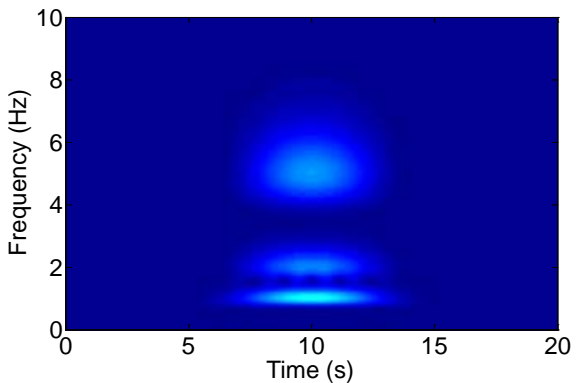

(d)

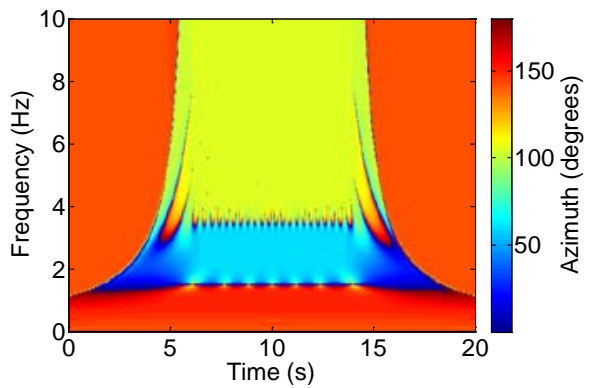

(b)

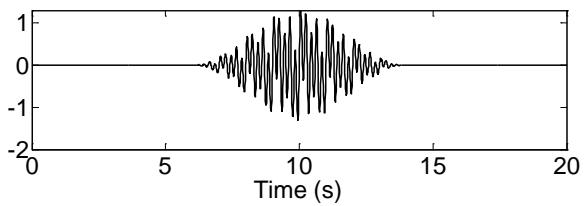

(c)

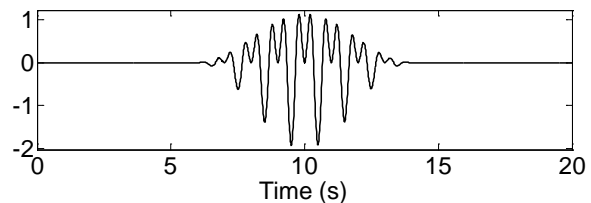

(b)

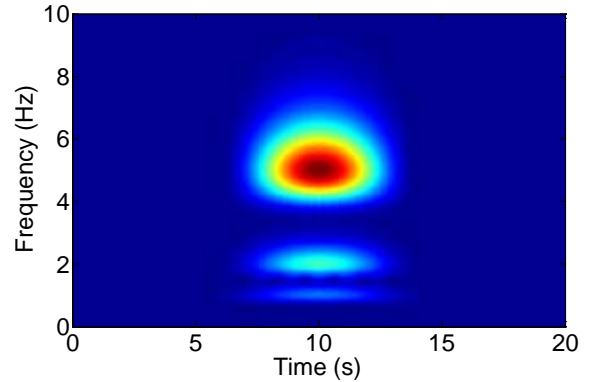

(e) (c)

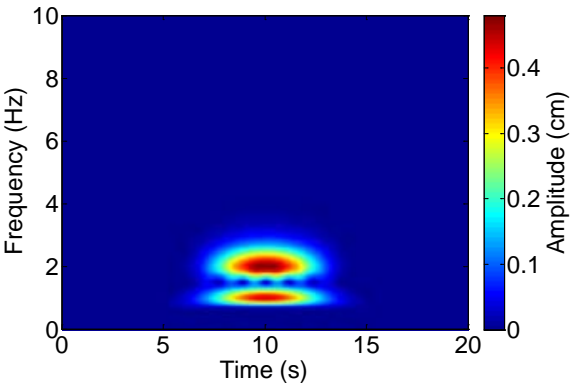

(f)

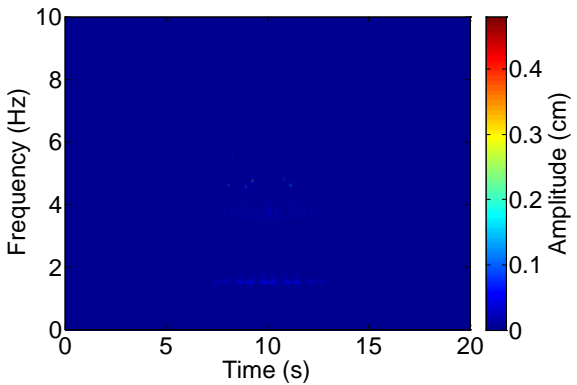

Figure 6. Time-frequency representation of synthetic signal. (a) Amplitude of North component, (b) Amplitude of East component, (c) Amplitude of vertical component, (d) Azimuth of direction of propagation according to Eq. (20), (e) Amplitude of radial component (f) Amplitude of transverse component. 
(a)

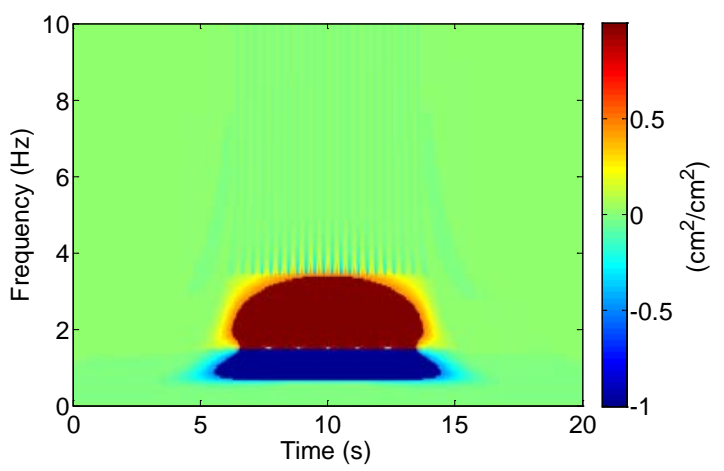

(c)

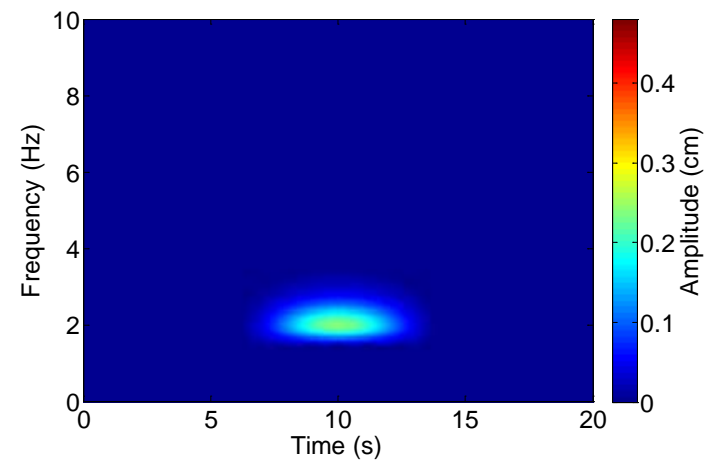

(b)

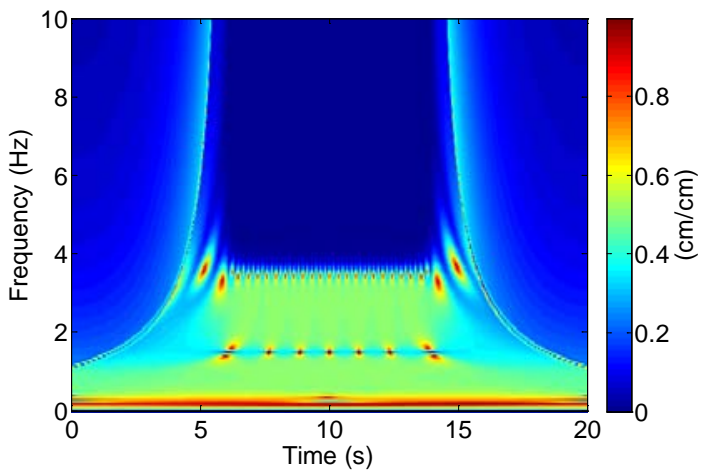

(d)

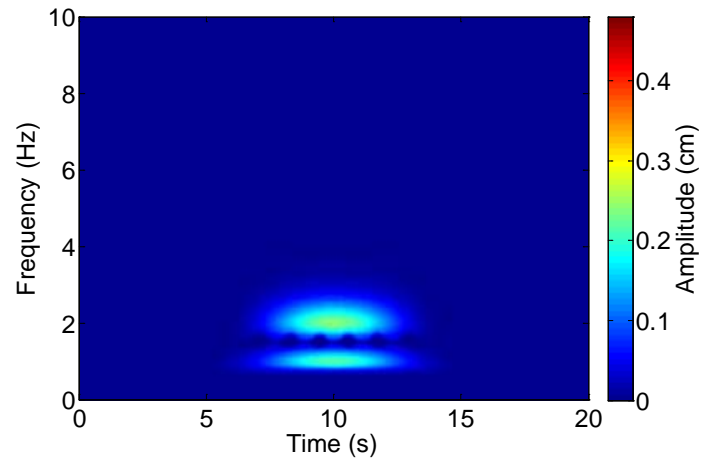

Figure 7. Comparison of filtering using the IRE and NIP criteria. (a) Normalized inner product of radial and phase advanced vertical component, (b) IRE computed with the three-components of the signal, (c) Radial displacement component filtered using the NIP criterion, (d) Radial displacement component filtered using the IRE criterion.

(a)

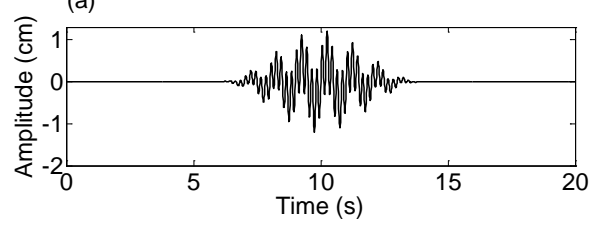

(d)

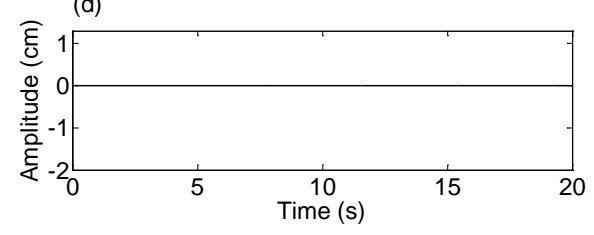

(b)

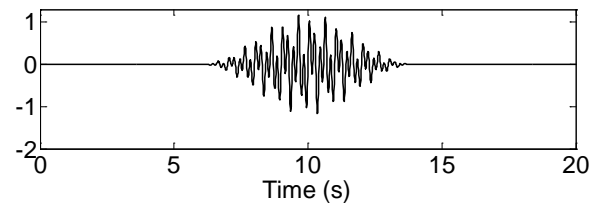

(e)

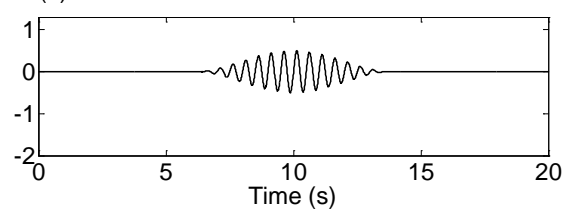

(c)

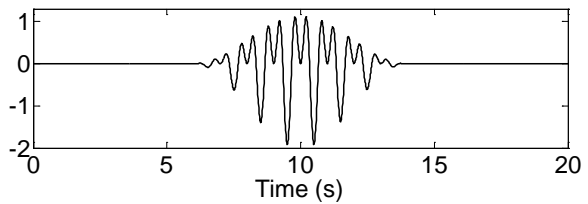

(f)

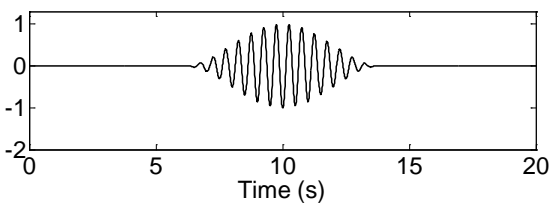

Figure 8. Rayleigh retro-grade wave extracted from synthetic signal. (a) Unfiltered transverse ( -) component, (b) Unfiltered radial ( -) component, (c) Unfiltered ( -) vertical component, (d) Extracted transverse component, (e) Extracted radial component, (f) Extracted vertical component. 


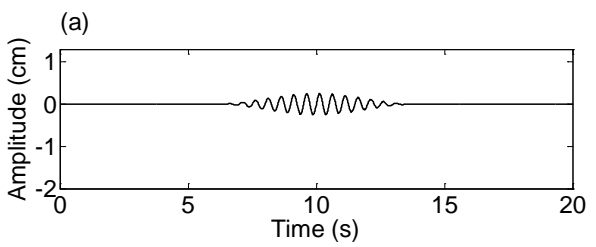

(b)

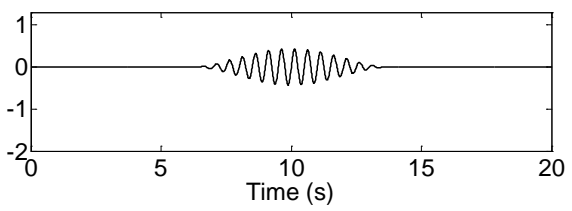

(c)

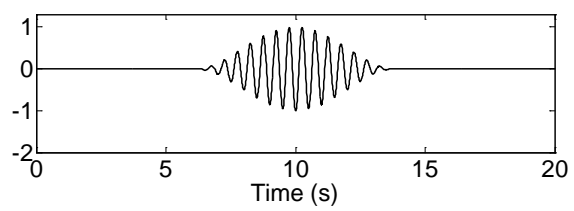

Figure 9. Extracted retro-grade Rayleigh wave. (a) North component, (b) East component, (c) Vertical component.

(a)

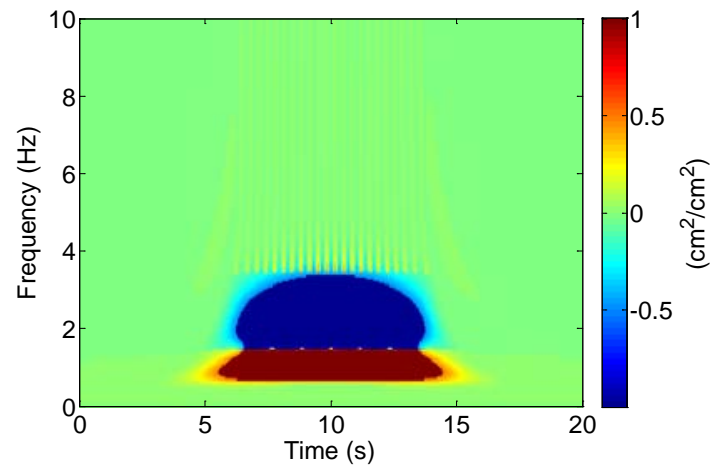

(b)

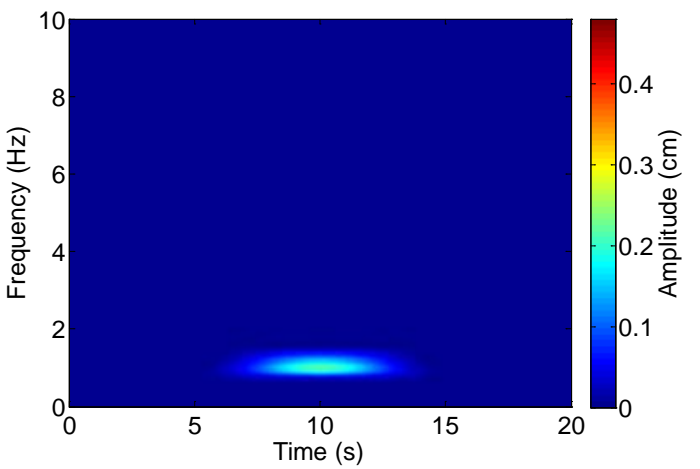

Figure 10. Extraction of pro-grade wave using the NIP criterion. (a) Normalized inner product of radial and phase delayed vertical component, (b) Amplitude of component filtered with the NIP criterion.

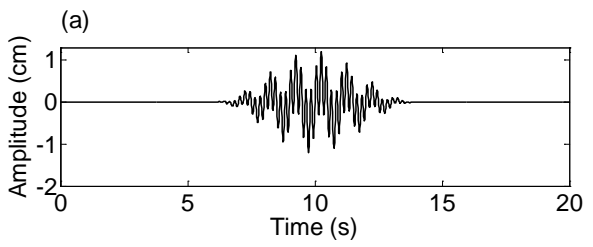

(b)
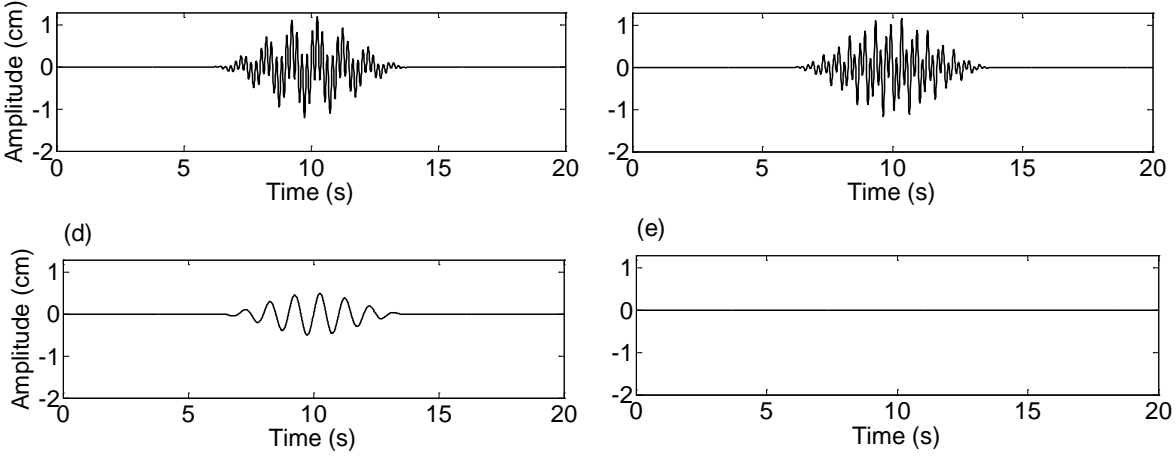

(e)

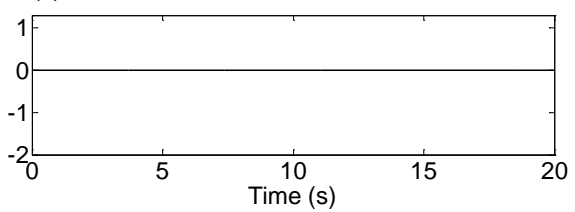

(c)

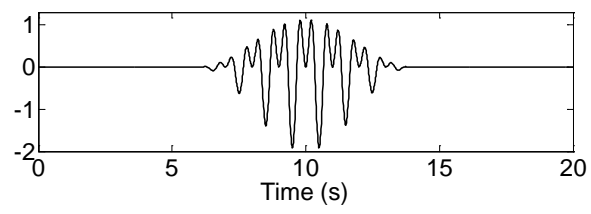

(f)

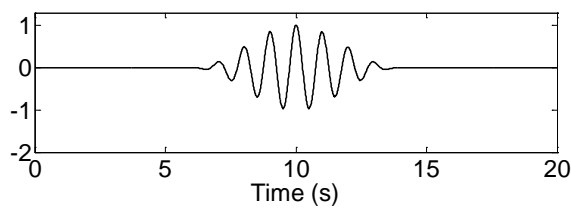

Figure 11. Rayleigh pro-grade wave extracted from synthetic signal. (a) Unfiltered radial ( -) component, (b) Unfiltered transverse ( -) component, (c) Unfiltered ( -) vertical component, (d) Extracted radial component, (e) Extracted transverse component, (f) Extracted vertical component.

(a)

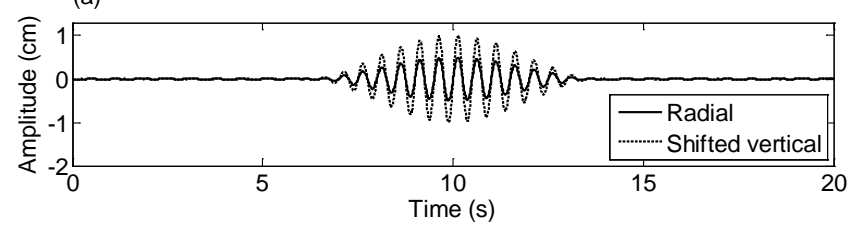

(b)

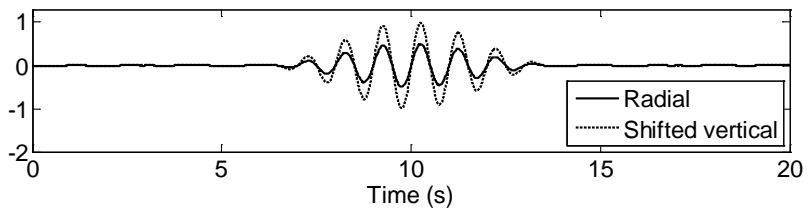

Figure 12. Comparison of radial and shifted vertical components of extracted waves. (a) Retro-grade wave (2 Hz), (b) Prograde wave $(1 \mathrm{~Hz})$. 
(a)

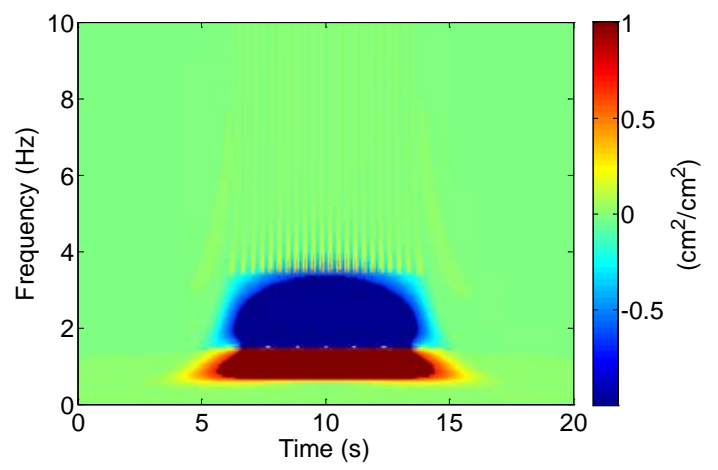

(b)

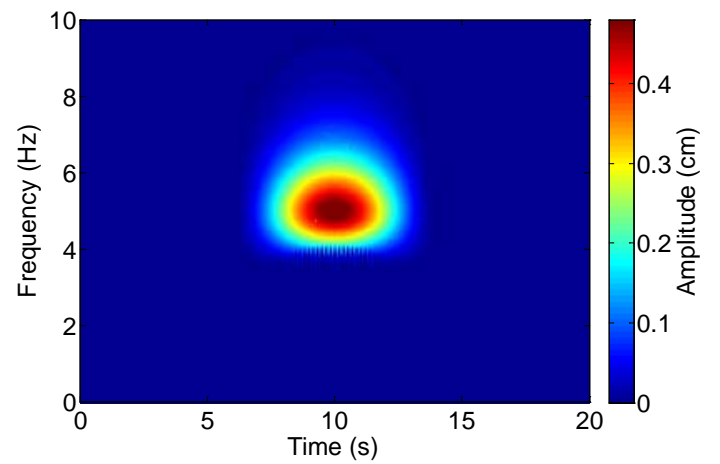

Figure 13. Extraction of linearly polarized wave using the NIP criterion. (a) Normalized inner product of radial and phase delayed vertical component, (b) Amplitude of component filtered with the NIP criterion.

(a)

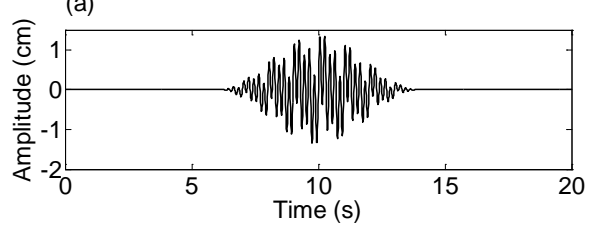

(d)

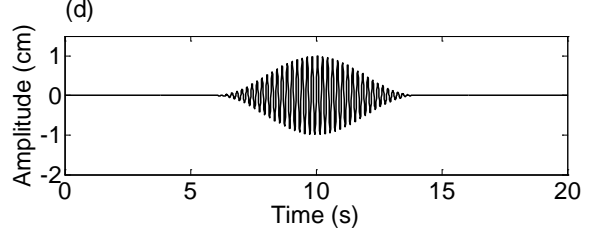

(b)

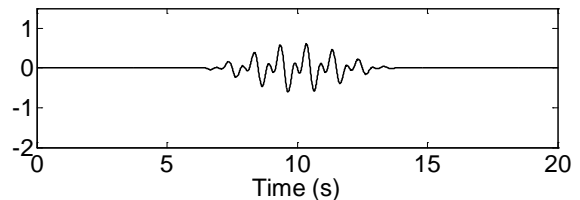

(e)

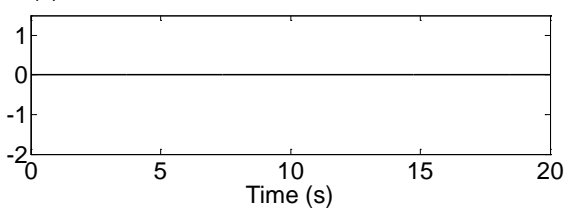

(c)

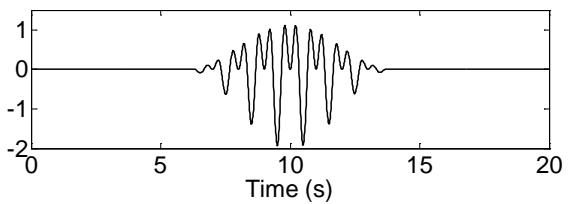

(f)

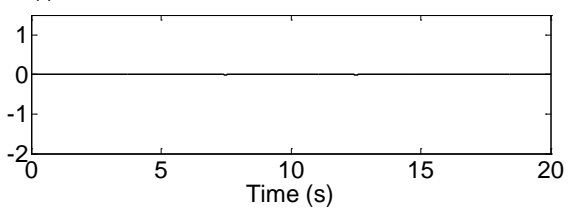

Figure 14. Linearly polarized wave extracted from synthetic signal. (a) Unfiltered radial component, (b) Unfiltered transverse component, (c) Unfiltered vertical component, (d) Extracted radial component, (e) Extracted transverse component, (f) Extracted vertical component. 


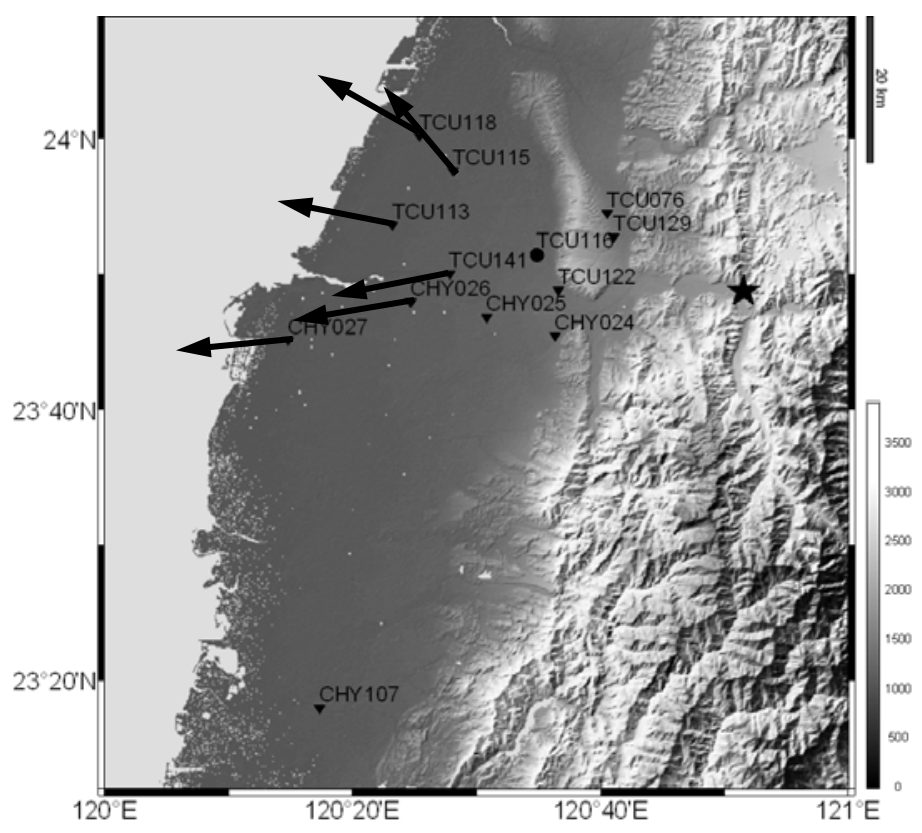

Figure 15. Map illustrating the location of the stations on the West Coastal Plain considered in this study. For some stations the arrows indicate the direction Rayleigh wave propagation estimated by Wang et al. (2006). The star indicates the location of the epicenter of the event. The black circle indicates the location of station TCU116.

(a)

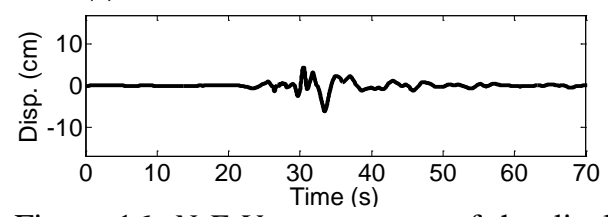

(b)

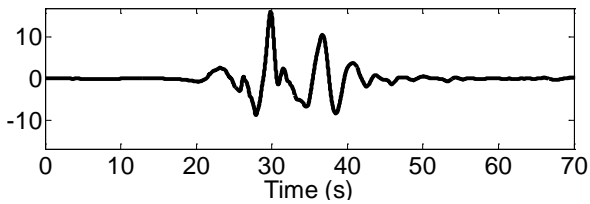

(c)

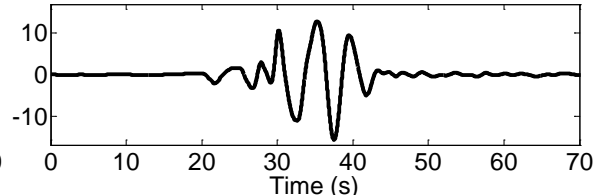

Figure 16. $N-E-V$ components of the displacement history (in $\mathrm{cm}$ ) for Chi-Chi aftershock 1803 at station TCU116. (a) North component (b) East component (c) Vertical component.

(a)

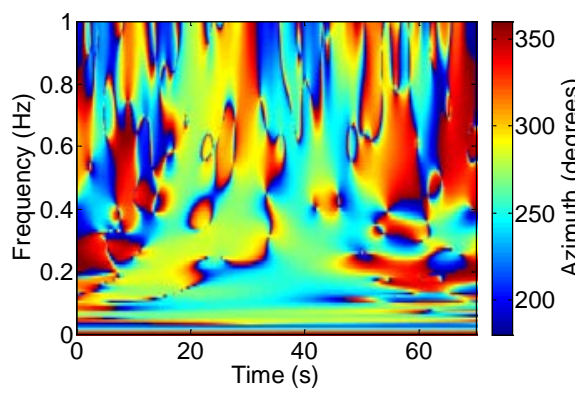

Figure 17. Radial and Transverse disp propagation, (b) Amplitude of (b)

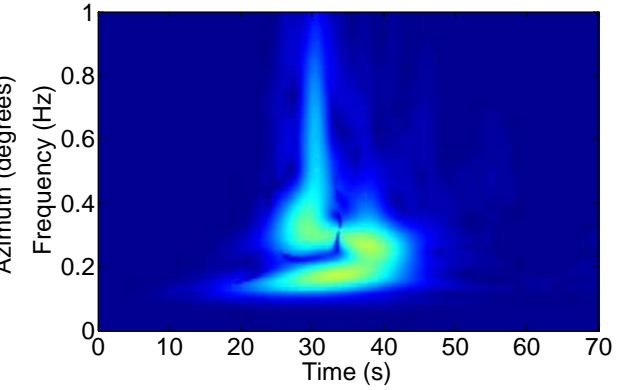

(c)

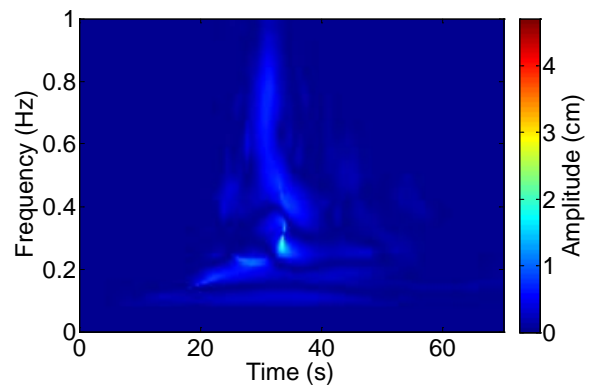

component (c) Amplitude of
component. 
(a)

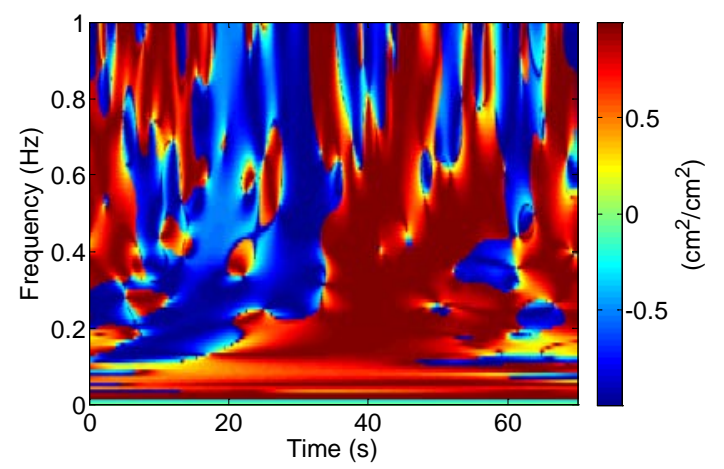

(c)

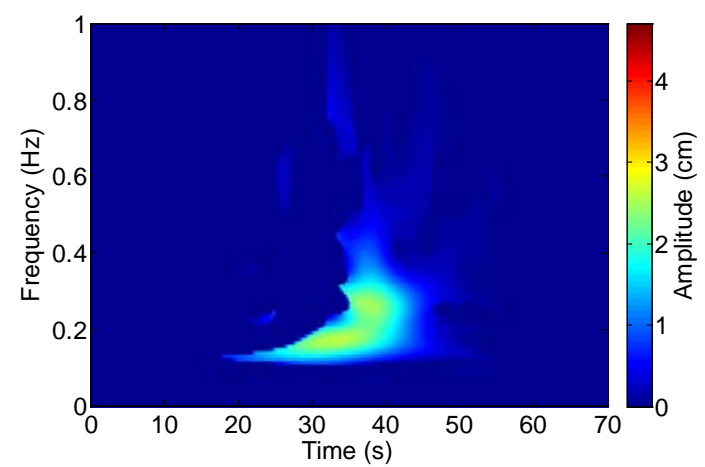

(b)

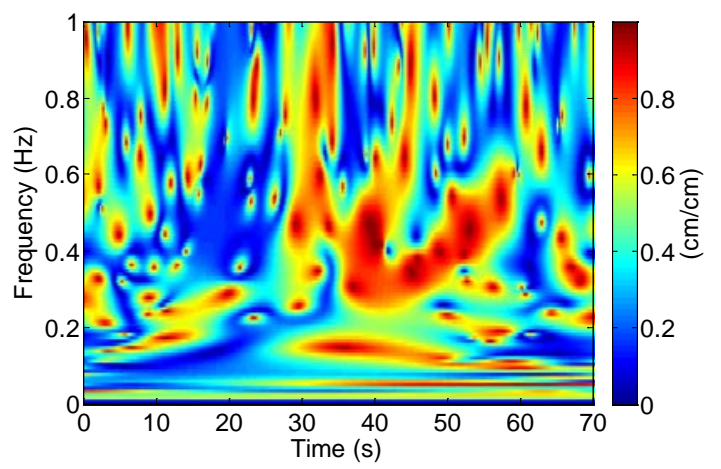

(d)

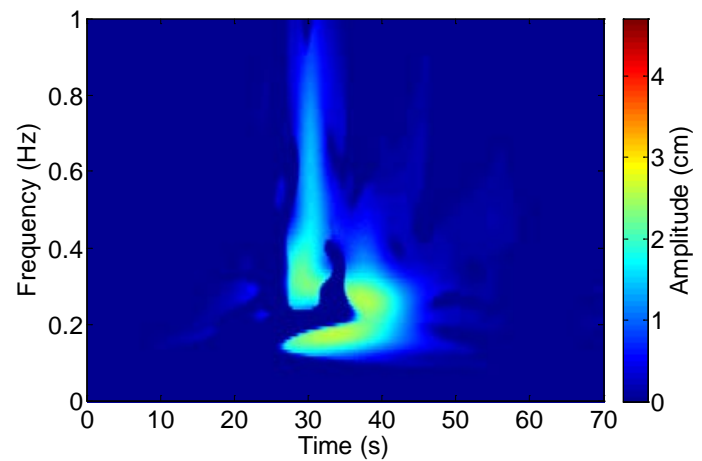

Figure 18. Comparison of filtering to extract the retro-grade wave from recording at station TCU116 using the IRE and NIP criteria. (a) Normalized inner product of and phase advanced component, (b) IRE computed with the threecomponents of the signal, (c) component filtered with the NIP criterion, (d) component filtered with the IRE criterion.

(a)

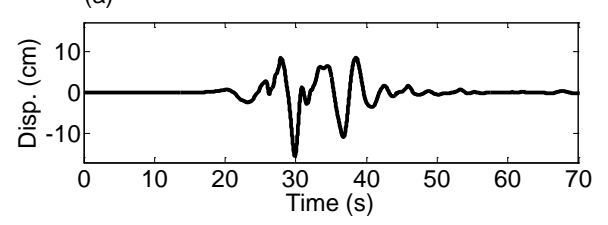

(d)

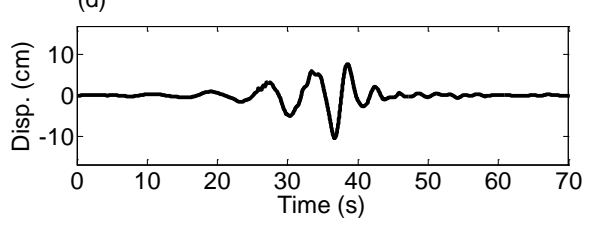

(b)

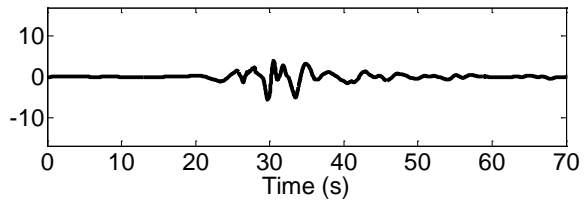

(e)

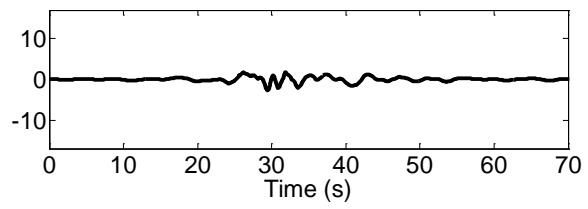

(c)

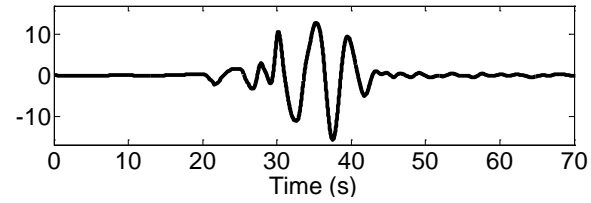

(f)

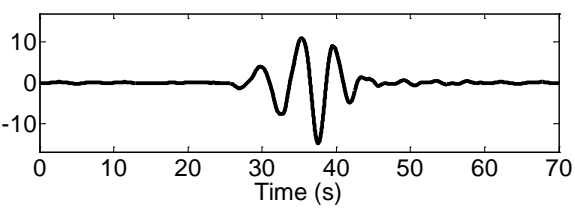

Figure 19. Rayleigh retro-grade wave extracted at station TCU116. (a) Unfiltered radial component, (b) Unfiltered transverse component, (c) Unfiltered vertical component, (d) Extracted radial component, (e) Extracted transverse component, (f) Extracted vertical component. 
(a)

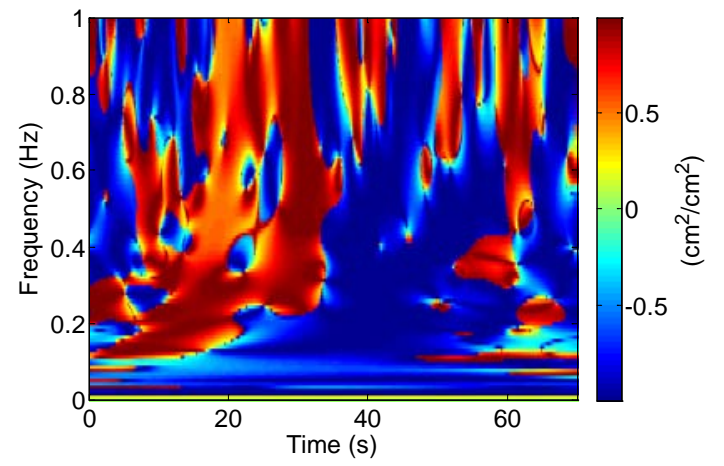

(b)

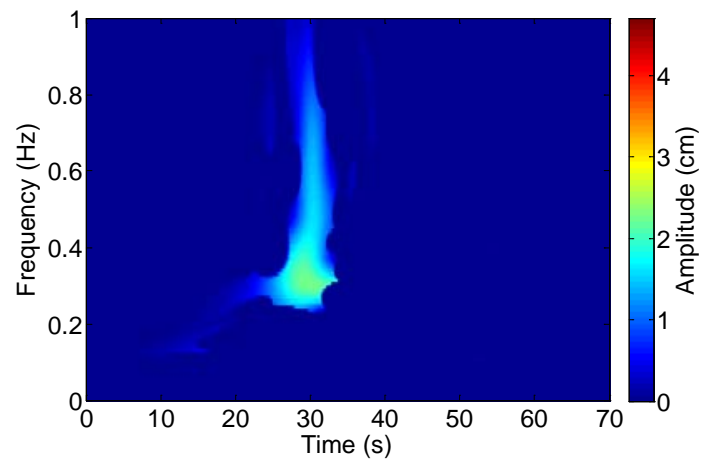

Figure 20. Extraction of pro-grade Rayleigh wave from recording at station TCU116. (a) Normalized inner product of and phase delayed component, (b) Amplitude of component filtered with the NIP criterion.

(a)

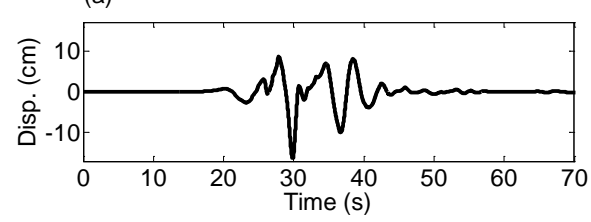

(d)

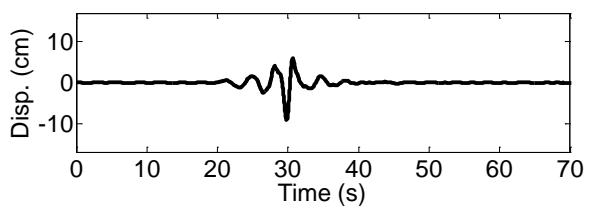

(b)

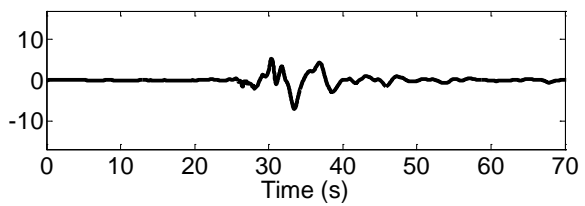

(e)

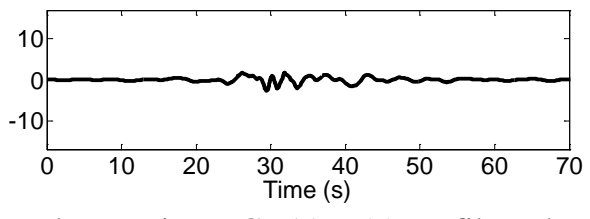

(c)

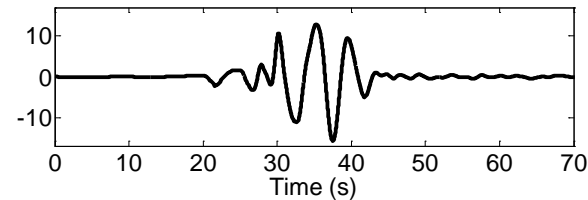

(f)

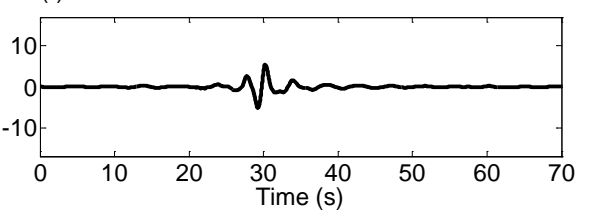

Figure 21. Rayleigh pro-grade wave extracted at station TCU116. (a) Unfiltered radial component, (b) Unfiltered transverse component, (c) Unfiltered vertical component, (d) Extracted radial component, (e) Extracted transverse component, (f) Extracted vertical component.

(a)

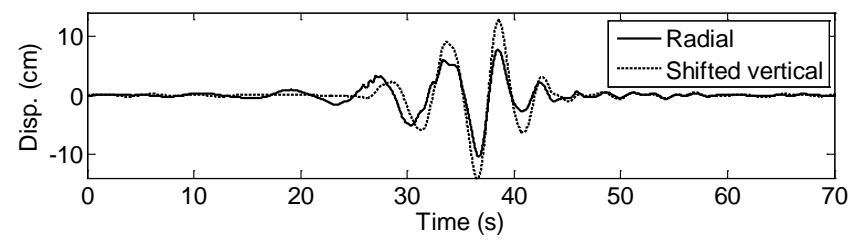

(b)

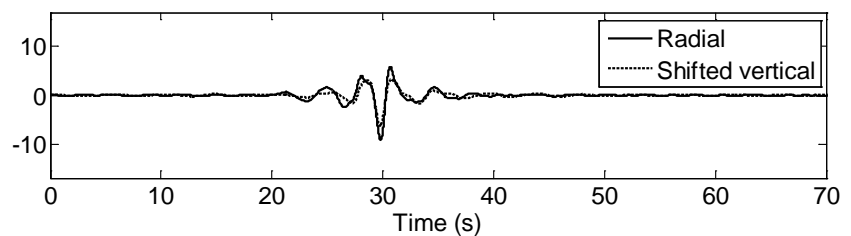

Figure 22. Comparison of radial and shifted vertical components of extracted Rayleigh waves at station TCU116. (a) Retrograde wave, (b) Pro-grade wave.

(a)

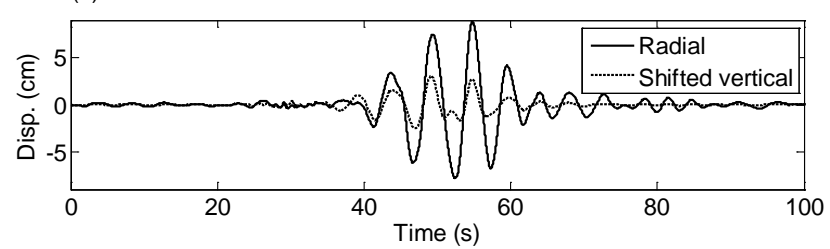

(b)

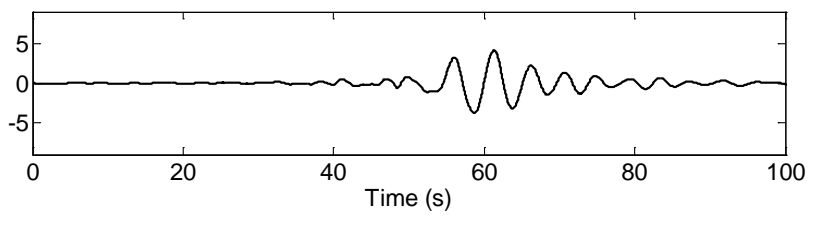

Figure 23. $R-T-V$ displacement components of extracted waves at station TCU118. (a) Comparison of radial and shifted vertical component of retro-grade Rayleigh wave (b) Extracted (Love) wave in transverse direction. 
(a)

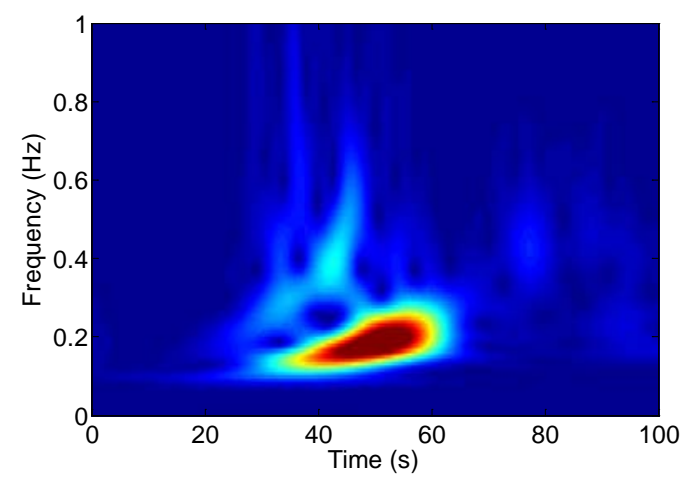

(b)

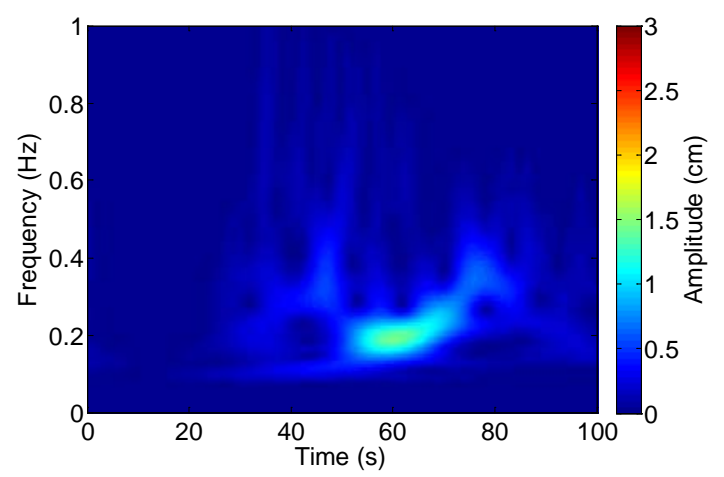

Figure 24. Radial and Transverse time-frequency components for unfiltered recording at station TCU118. (a) Amplitude of radial component (b) Amplitude of transverse component.
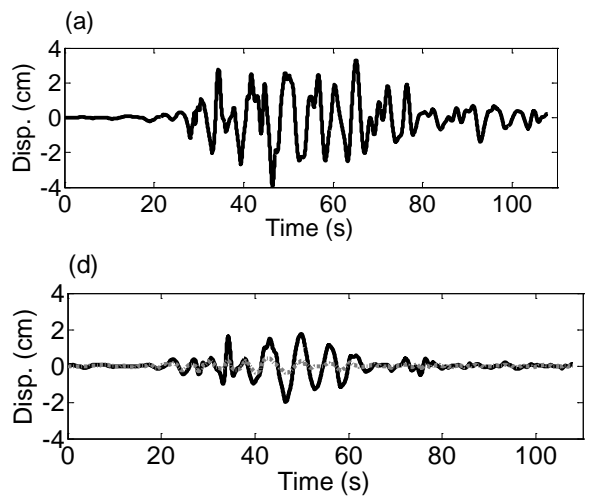
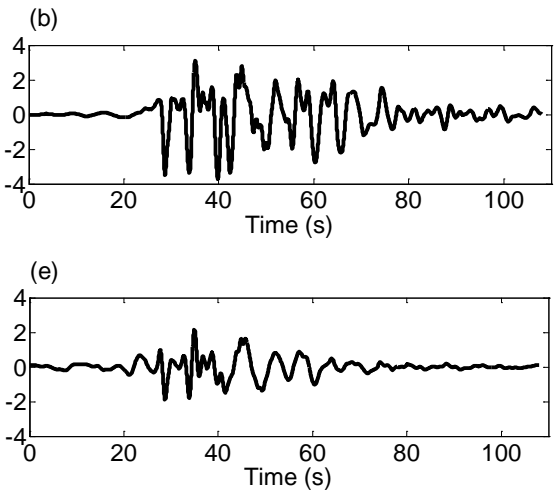
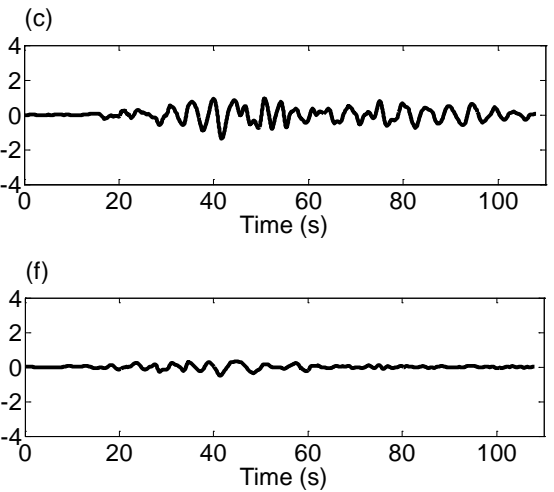

Figure 25. Extracted waves from recording at station CHY107. (a) Unfiltered radial component, (b) Unfiltered transverse component, (c) Unfiltered vertical component, (d) Extracted radial component (black line) and shifted vertical component (gray line), (e) Extracted transverse component, (f) Extracted vertical component. 
Station

TCU129
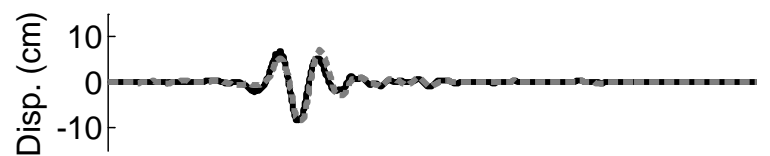

Azimuth: 293.757 degrees

Azimuth: 290.9743 degrees

TCU076

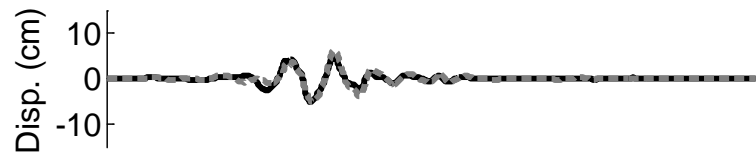

Azimuth: 318.8572 degrees

TCU122

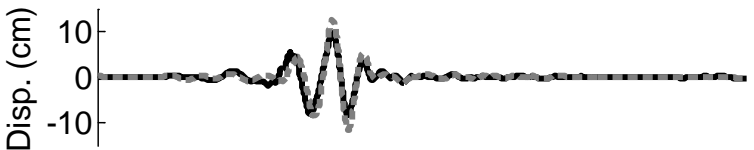

Azimuth: 265.8207 degrees

CHY024

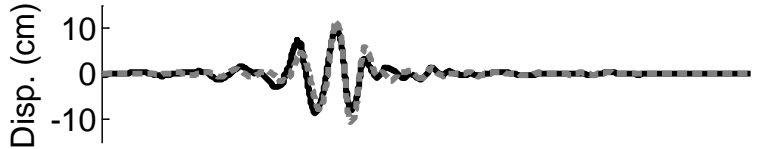

Azimuth: 246.5196 degrees

CHY025

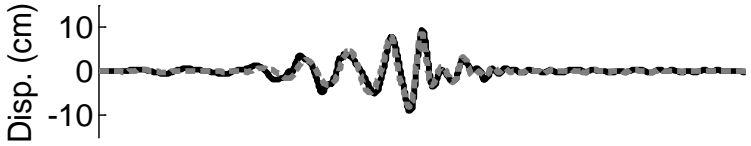

Azimuth: 294.2067 degrees

TCU115

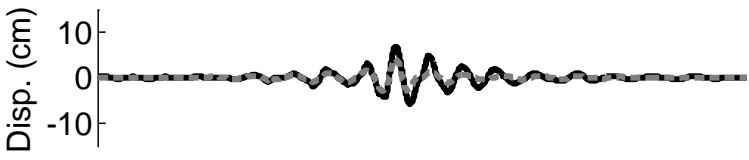

Azimuth: 307.6878 degrees

TCU141

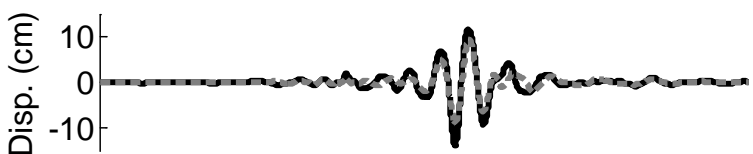

Azimuth: 254.5216 degrees

CHY026

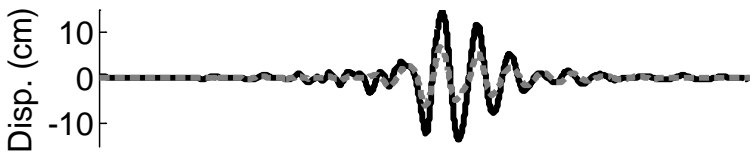

Azimuth: 259.9459 degrees

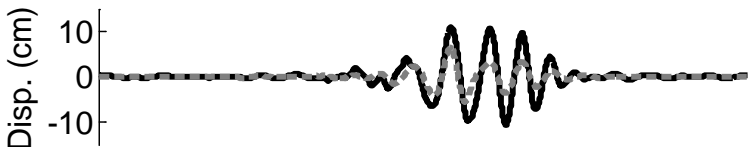

Azimuth: 279.9617 degrees
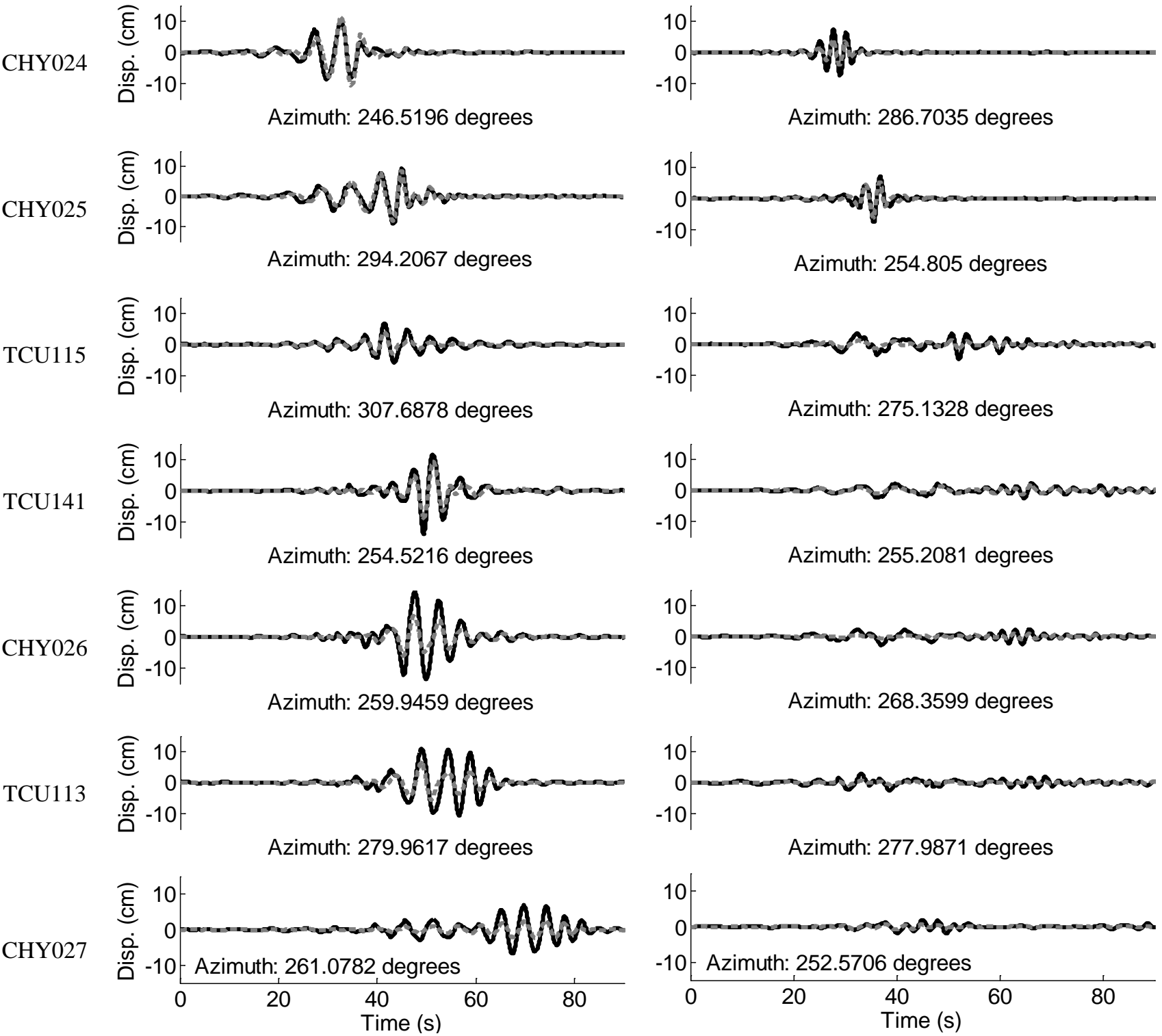

Azimuth: 286.7035 degrees

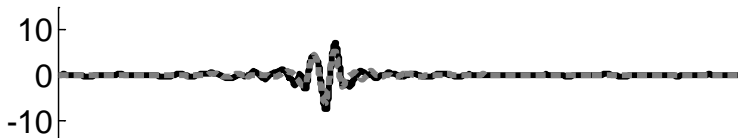

Azimuth: 254.805 degrees

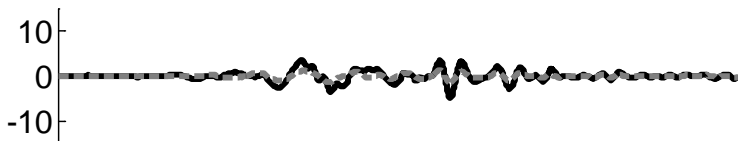

Azimuth: 275.1328 degrees

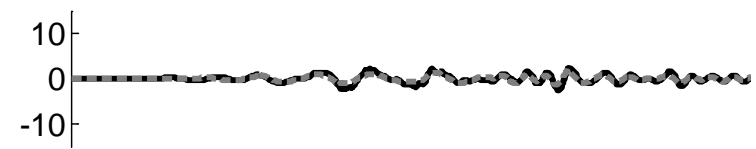

Azimuth: 255.2081 degrees

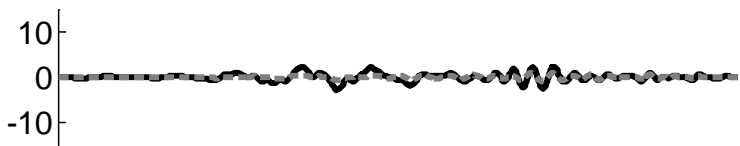

Azimuth: 268.3599 degrees

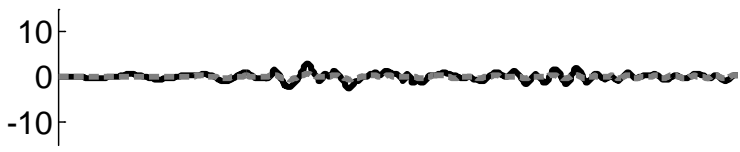

Azimuth: 277.9871 degrees

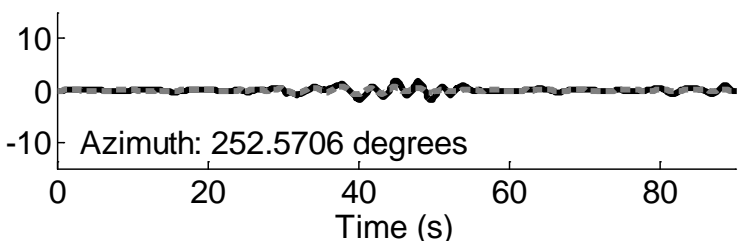

Figure 26. Radial component (black solid line) and shifted vertical component (gray dashed line) of extracted Rayleigh waves at different stations of the WCP plain in Taiwan. (a) Retro-grade waves, (b) Pro-grade waves. 
Station

TCU129

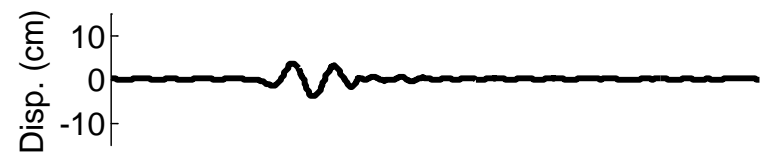

TCU076
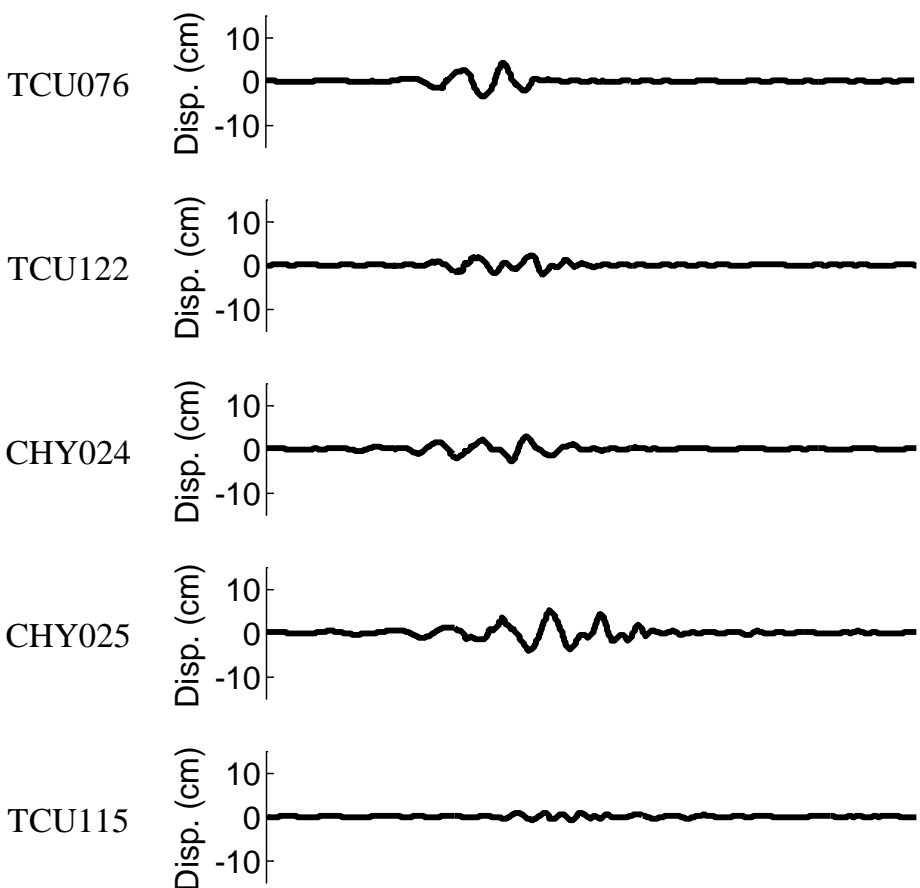

TCU141

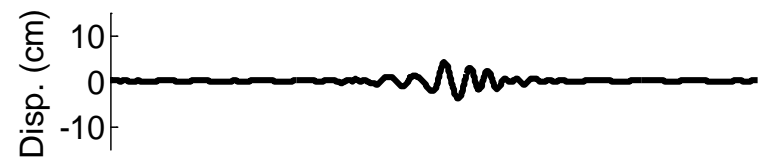

CHY026

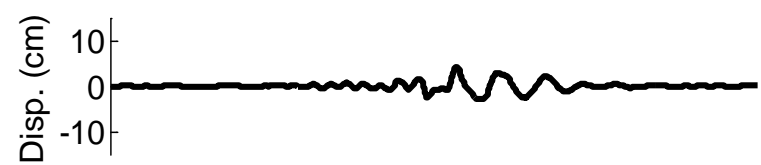

TCU113

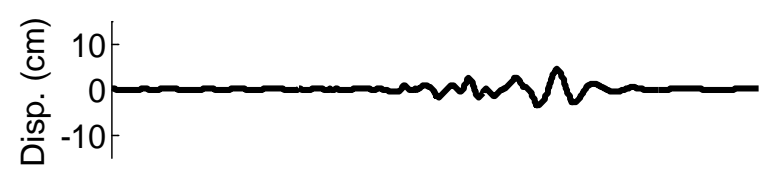

CHY027

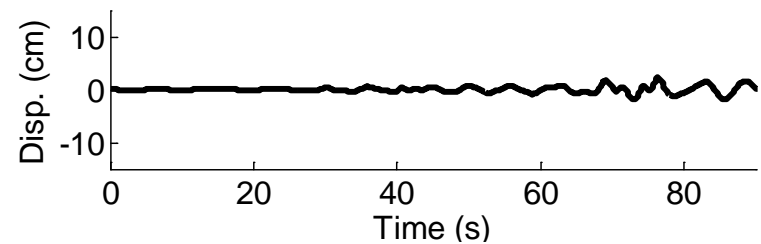

Figure 27. Extracted Love waves at different stations of the WCP plain in Taiwan. 